\title{
Mixed Convection Heat Transfer for Nanofluids in a Lid-Driven Shallow Rectangular Cavity Uniformly Heated and Cooled from the Vertical Sides: The Opposing Case
}

\author{
Hassan El Harfi ${ }^{1}$, Mohamed Naïmi ${ }^{1 *}$, Mohamed Lamsaadi ${ }^{2}$, \\ Abdelghani Raji ${ }^{1}$, Mohammed Hasnaoui ${ }^{3}$ \\ ${ }^{1}$ Physics Department, Faculty of Sciences and Technologies, Sultan Moulay Slimane University, \\ Laboratory of Flows and Transfers Modelling (LAMET), Beni-Mellal, Morocco \\ ${ }^{2}$ Polydisciplinary Faculty, Sultan Moulay Slimane University, Interdisciplinary Laboratory of \\ Research in Sciences and Technologies (LIRST), Beni-Mellal, Morocco \\ ${ }^{3}$ Physics Department, Faculty of Sciences Semlalia, Cadi Ayyad University, Laboratory of Fluid \\ Mechanics and Energetics (LMFE), Marrakech, Morocco \\ Email: "naimi@fstbm.ac.ma; ${ }^{*}$ naimima@yahoo.fr
}

Received May 31, 2013; revised July 1, 2013; accepted July 10, 2013

Copyright (C) 2013 Hassan El Harfi et al. This is an open access article distributed under the Creative Commons Attribution License, which permits unrestricted use, distribution, and reproduction in any medium, provided the original work is properly cited.

\begin{abstract}
An investigation on flow and heat transfer due to mixed convection, in a lid-driven rectangular cavity filled with $\mathrm{Cu}$ water nanofluids and submitted to uniform heat flux along with its vertical short sides, has been conducted numerically by solving the full governing equations with the finite volume method and the SIMPLER algorithm. In the case of a slender enclosure, these equations are considerably reduced by using the parallel flow concept. Solutions, for the flow and temperature fields, and the heat transfer rate, have been obtained depending on the governing parameters, which are the Reynolds, the Richardson numbers and the solid volume fraction of nanoparticles. A perfect agreement has been found between the results of the two approaches for a wide range of the abovementioned parameters. It has been shown that at low and high Richardson numbers, the convection is ensured by lid and buoyancy-driven effects, respectively,

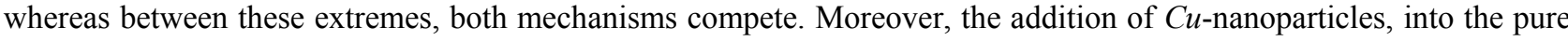
water, has been seen enhancing and degrading heat transfer by lid and buoyancy-driven effects, respectively.
\end{abstract}

Keywords: Component Nanofluids; Mixed Convection; Heat Transfer; Lid-Driven Cavity; Parallel Flow Assumption; Finite Volume Method

\section{Introduction}

Heat transfer in fluidic systems has often been the subject of ambitious research in order to enhance it considering its importance in several industrial processes. However, with conventional fluids, such as water and oil, whose thermal conductivity is inherently poor, heat transfer is limited, which is a crucial problem to challenge. Also, current design solutions already push available technology to its limits, and an innovative way should be taken. In such a context, Choi of Argonne National Laboratory [1] developed the novel concept of nanofluids as a route to improve the performances of heat transfer fluids currently available. This new class of advanced heat transfer fluids is engineered by dispersing solid nanoparticles

"Corresponding author. (metallic, non-metallic or polymeric), smaller than 100 $\mathrm{nm}$ in diameter, in base fluids (aqueous or organic host liquids), which confers a large thermal conductivity on these ones and makes them potentially useful in engineering equipments involving heat transfer. To know about nanoparticles, nanofluids, their production and applications, see, for instance, the report of $\mathrm{Yu}$ et al. is currently available in [2].

During the last decade, nanofluids have attracted lots of researchers, who are encouraged by their critical importance and promising role, as new advanced heat transfer fluids, to take up challenges. Therefore, numerous studies, on convection heat transfer, have been conducted, and most of them have dealt with forced convection, indicating that nanoparticle suspensions have unquestionably a great potential for heat transfer enhancement, as reported 
in a recent paper by Corcione [3]. In contrast, although the investigations concerned with buoyancy-driven convection are relatively few, they have seen a gradual increase lately, leading to contradictory findings, thus leaving still unanswered question, if the use of nanoparticle suspensions for natural convection applications is actually advantageous with respect to pure liquids [3]. At the same time, mixed convection has not received either less attention in view of the number of the related works recently done. Among them, flow and heat transfer problem in lid-driven cavities, which finds applications in industrial processes such as food processing, float glass production [4], thermal-hydraulics of nuclear reactors [5], dynamics of lakes [6], crystal growth, flow and heat transfer in solar ponds [7], lubrication technologies [8] and so on. The interaction of the shear driven flow due to the lid motion and natural convective flow due to the buoyancy effect are quite complex, which necessitates a comprehensive analysis to understand the physics of the resulting flow and heat transfer process. In this respect, different configurations and combinations of thermal and dynamical boundary conditions have been considered and analyzed by some investigators. The contributions can be divided in two cases:

1) Steady state case where all boundary conditions are time independent. In this regard, it is advisable to mention the work of Tiwari and Das [8], who studied heat transfer enhancement in a nanofluid-filled square cavity, with the vertical sides moving and differentially heated, while the horizontal ones are insulated and motionless. Three situations, depending on the direction of the moving walls, were examined, and a model taking into account the solid volume fraction of nanoparticles was developed to analyze the nanofluids behavior. With only one uniformly moving wall, from left to right, first, it is to bring up the research of Abu-Nada and Chamkha [9] deal with mixed convection flow in an inclined square enclosure filled with a nanofluid. The left and right walls are kept insulated while the bottom and the moving top ones are maintained at constant cold and hot temperatures, respectively. It was found that significant heat transfer enhancement can be obtained due to the presence of nanoparticles and that this is accentuated by inclination of the enclosure at moderate and large Richardson numbers. Mahmoodi [10] investigated mixed convection fluid flow and heat transfer in rectangular enclosures filled with a nanofluid. The left and right walls as well as the top one are maintained at a constant cold temperature. The moving bottom is kept at a constant hot temperature. A parametric study was performed and the effects of the Richardson number, the aspect ratio of the enclosure and the volume fraction of the nanoparticles on the fluid flow and heat transfer were analysed. It was found that for the selected values of the Richardson number, the average Nusselt number increases with the nanoparticles volume fraction, and seems to be higher with tall enclosures than with shallow ones. In the case of a nanofluid-filled square cavity with cold sides, a partially heated (with constant heat flux heater) and insulated bottom, and a moving cold top, Mansour et al. [11] examined the effects of Reynolds number, type of nanofluids, size and location of the heater and the volume fraction of the nanoparticles in their study related to mixed convection. They observed that the heat transfer enhances with all the above mentioned parameters. Muthtamilselvan et al. [12] studied heat transfer enhancement of nanofluids in rectangular enclosures, where the moving top is at higher constant temperature than the bottom, whereas the left and right boundaries are insulated. They found that at higher aspect ratios, the heat transfer rate increases strongly with the nanoparticles volume fraction. Nemati et al. [13] investigated heat transfer performance of a moving top square cavity, filled with nanofluids and subject to different side wall temperatures. They reported that an increase of nanoparticles volume fraction enhances heat transfer, but such an effect reduces with the Reynolds number. As for Talebi et al. [14], they conducted an investigation on mixed convection flows in a square liddriven cavity, having left and right sides heated and cooled, respectively, and moving top and bottom both adiabatic, utilizing nanofluids. These authors showed that, at given Rayleigh and Reynolds numbers, an increase of the nanoparticles concentration favours the flow and heat transfer. Finally, like Tiwari and Das [8], Sheikhzadeh et al. [15] were interested in laminar mixed convection of a nano-fluid in two sided lid-driven enclosures. The moving left and right walls are maintained at constant cold and hot temperatures, respectively, while the horizontal ones are insulated. The effect of moving direction of walls on mixed convection is studied for various Richardson numbers, aspect ratios and nanoparticles volume fractions, and was found to affect mainly the flow field, temperature gradient and heat transfer. In addition, increasing the volume fraction of nanoparticles resulted in a linear increase of the average Nusselt number, as an index of heat transfer rate improvement, for all the considered cases.

2) Unsteady state case, where some boundary conditions are time dependent as in the only work done, in this subject, by Karimipour et al. [16], where periodic mixed convection of a nanofluid inside a rectangular cavity, with insulted vertical sides and hot temperature bottom kept at rest and cold temperature top horizontally oscillating, was carried out. The effects of Richardson number and volume fraction of nanoparticles on the flow and thermal behaviour of the nanofluid were examined. It was observed that the best heat transfer is obtained with a Richardson number lower than unit and that the higher value of this parameter corresponds to the lower amplitude of the oscillation of the heat transfer rate in the 
steady periodic state. In addition, heat transfer was found to be improved by nanoparticles presence.

All the above mentioned studies are of numerical nature, using a finite volume method (for the most), a finite difference method or Lattice Boltzmann method to solve the governing equations and various single-phase models to describe effective conductivity and viscosity of the considered nanofluids, which are principally $\mathrm{Al}_{2} \mathrm{O}_{3}$ or $\mathrm{Cu}$-water.

To the best of our knowledge, the problem of mixed convection heat transfer of nanofluids in a lead-driven enclosure subject to Neumann boundary conditions for temperature (i.e. boundaries subject to heat fluxes) is not yet analyzed. So, in order to know more about the effect of the boundary conditions kind on flow and heat transfer within nanofluids, the present paper focuses on such a problem within a two-dimensional shallow rectangular enclosure, filled with $\mathrm{Cu}$-water nanofluids, whose short vertical sides are submitted to uniform heat fluxes while the long horizontal ones are maintained adiabatic with the top moving in the opposite direction to the heat flux. A numerical solution of the full governing equations is obtained via a finite volume method. An analytical one, based on the parallel flow approximation, is also proposed. The results are presented, in terms of streamlines, isotherms, stream function and temperature profiles and heat transfer rates, and discussed for various values of the dimensionless parameters, controlling the problem, which are the Reynolds, $R e$, and Richardson, $R i$, numbers, and the solid volume fraction of nanoparticles, $\Phi$.

\section{Mathematical Formulation}

The studied configuration is sketched in Figure 1. It is a shallow rectangular enclosure of height $H^{\prime}$ and length $L^{\prime}$, filled with $\mathrm{Cu}$-water nanofluids. The long horizontal walls are adiabatic, while the vertical short ones are submitted to a uniform density of heat flux, $q^{\prime}$. All these boundaries are rigid, impermeable and motionless apart from the top one which moves in its own plane from right to left at uniform velocity, $U_{0}^{\prime}$. The main assumptions made here are those commonly used, i.e.:

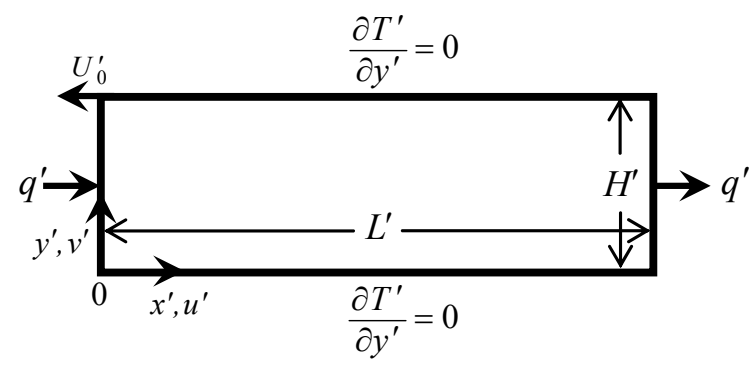

Figure 1. Schematic view of the geometry and coordinates system.
The base fluid and the nanoparticles are in thermal equilibrium and they flow at the same velocity (i.e. no slip occurs between them or the nanoparticles are uniformly dispersed within the base fluid so that the resulting nanofluid can be considered a single-phase fluid).

- The nanoparticles are spherical;

- The nanofluid is Newtonian and incompressible;

- The thermophysical properties of the considered nanofluids are constant (taken at the reference temperature, $T_{0}^{\prime}$ ) except for the density in the buoyancy term (containing the gravitational acceleration, $g$ ), which obeys the Boussinesq approximation;

- The flow is two-dimensional, laminar and steady;

- The radiation heat transfer between the sides of the cavity is negligible when compared with the other mode of heat transfer.

Therefore, the equations describing the conservation of mass (1), momentum (2)-(3) and energy (4), written in terms of velocity components $\left(u^{\prime}, v^{\prime}\right)$, pressure $\left(p^{\prime}\right)$ and temperature $\left(T^{\prime}\right)$, are:

$$
\begin{gathered}
\frac{\partial u^{\prime}}{\partial x^{\prime}}+\frac{\partial v^{\prime}}{\partial y^{\prime}}=0 \\
\frac{\partial u^{\prime}}{\partial t^{\prime}}+u^{\prime} \frac{\partial u^{\prime}}{\partial x^{\prime}}+v^{\prime} \frac{\partial u^{\prime}}{\partial y^{\prime}}=-\frac{1}{\rho_{n f}} \frac{\partial P^{\prime}}{\partial x^{\prime}}+\frac{\mu_{n f}}{\rho_{n f}}\left(\frac{\partial^{2} u^{\prime}}{\partial x^{\prime 2}}+\frac{\partial^{2} u^{\prime}}{\partial y^{\prime 2}}\right) \\
\frac{\partial v^{\prime}}{\partial t^{\prime}}+u^{\prime} \frac{\partial v^{\prime}}{\partial x^{\prime}}+v^{\prime} \frac{\partial v^{\prime}}{\partial y^{\prime}} \\
=-\frac{1}{\rho_{n f}} \frac{\partial P^{\prime}}{\partial y^{\prime}}+\frac{\mu_{n f}}{\rho_{n f}}\left(\frac{\partial^{2} v^{\prime}}{\partial x^{\prime 2}}+\frac{\partial^{2} v^{\prime}}{\partial y^{\prime 2}}\right)+\frac{1}{\rho_{n f}}(\rho \beta)_{n f} g\left(T^{\prime}-T_{0}^{\prime}\right) \\
\frac{\partial T^{\prime}}{\partial t^{\prime}}+u^{\prime} \frac{\partial T^{\prime}}{\partial x^{\prime}}+v^{\prime} \frac{\partial T^{\prime}}{\partial y^{\prime}}=\alpha_{n f}\left(\frac{\partial^{2} T^{\prime}}{\partial x^{\prime 2}}+\frac{\partial^{2} T^{\prime}}{\partial y^{\prime 2}}\right)
\end{gathered}
$$

To close the problem, the following appropriate boundary conditions are applied:

$$
\begin{gathered}
u^{\prime}=v^{\prime}=0 \text { and } \frac{\partial T^{\prime}}{\partial x^{\prime}}+\frac{q^{\prime}}{k_{n f}}=0 \text { for } x^{\prime}=0 \text { and } x^{\prime}=L^{\prime} \\
u^{\prime}=v^{\prime}=0 \text { and } \frac{\partial T^{\prime}}{\partial y^{\prime}}=0 \text { for } y^{\prime}=0 \\
u^{\prime}+U_{0}^{\prime}=v^{\prime}=0 \text { and } \frac{\partial T^{\prime}}{\partial y^{\prime}}=0 \text { for } y^{\prime}=H^{\prime}
\end{gathered}
$$

To model the effective physical properties of the nanofluid, appearing in the above equations, the following formulas are used:

$$
\rho_{n f}=(1-\Phi) \rho_{f}+\Phi \rho_{n p}
$$

for the effective density, as shown in [2]; 


$$
\mu_{n f}=\frac{\mu_{f}}{(1-\Phi)^{2.5}}
$$

for the effective dynamic viscosity, which is due to Brinkman [2];

$$
(\rho \beta)_{n f}=(1-\Phi)(\rho \beta)_{f}+\Phi(\rho \beta)_{n p}
$$

for the thermal expansion coefficient [17];

$$
(\rho C p)_{n f}=(1-\Phi)(\rho C p)_{f}+\Phi(\rho C p)_{n p}
$$

for the heat capacity [2];

$$
\frac{k_{n f}}{k_{f}}=\frac{k_{n p}+2 k_{f}-2 \Phi\left(k_{f}-k_{n p}\right)}{k_{n p}+2 k_{f}+\Phi\left(k_{f}-k_{n p}\right)}
$$

for the effective thermal conductivity, due to MaxwellGarnett, which is a restriction of the Hamilton-Crosser model to spherical nanoparticles [2];

$$
\alpha_{n f}=\frac{k_{n f}}{(\rho C p)_{n f}}
$$

for the thermal diffusivity [18].

Note that the Subscripts $f, n f$ and $n p$ stand for the base fluid, nanofluid and nanoparticles, respectively.

On the other hand, using the characteristic scales $H^{\prime}$, $\rho_{f} U_{0}^{\prime 2}, H^{\prime} / U_{0}^{\prime}, U_{0}^{\prime}$, and $q^{\prime} H^{\prime} / k_{f}$, corresponding to length, pressure, time, velocity and temperature, respectively, the dimensionless governing equations and the corresponding boundary conditions are:

$$
\begin{gathered}
\frac{\partial u}{\partial x}+\frac{\partial v}{\partial y}=0 \\
\frac{\partial u}{\partial t}+u \frac{\partial u}{\partial x}+v \frac{\partial u}{\partial y}=-\frac{1}{\bar{\rho}} \frac{\partial P}{\partial x}+\frac{\bar{v}}{\operatorname{Re}}\left(\frac{\partial^{2} u}{\partial x^{2}}+\frac{\partial^{2} u}{\partial y^{2}}\right) \\
\frac{\partial v}{\partial t}+u \frac{\partial v}{\partial x}+v \frac{\partial v}{\partial y} \\
=-\frac{1}{\bar{\rho}} \frac{\partial P}{\partial y}+\frac{\bar{v}}{\operatorname{Re}}\left(\frac{\partial^{2} v}{\partial x^{2}}+\frac{\partial^{2} v}{\partial y^{2}}\right)+\frac{\bar{\beta}}{\bar{\rho}} R i T \\
\frac{\partial T}{\partial t}+u \frac{\partial T}{\partial x}+v \frac{\partial T}{\partial y}=\frac{\bar{\alpha}}{P e}\left(\frac{\partial^{2} T}{\partial x^{2}}+\frac{\partial^{2} T}{\partial y^{2}}\right) \\
u=v=\frac{\partial T}{\partial x}+\frac{1}{\bar{k}}=0 \text { for } x=0 \text { and } A \\
u=v=\frac{\partial T}{\partial y}=0 \text { for } y=0 \\
u+1=v=\frac{\partial T}{\partial y}=0 \text { for } y=1
\end{gathered}
$$

where $\bar{k}=k_{n f} / k_{f}, \quad \bar{\alpha}=\alpha_{n f} / \alpha_{f}, \quad \bar{v}=v_{n f} / v_{f}$, $\bar{\beta}=(\rho \beta)_{n f} /(\rho \beta)_{f}$ and $\bar{\rho}=\rho_{n f} / \rho_{f}$ are parameters depending on $\Phi$, according to models given above. In addition, to analysis the flow structure, the stream function, $\psi$, related to the velocity components via

$$
u=\frac{\partial \psi}{\partial y} \text { and } v=-\frac{\partial \psi}{\partial x}(\text { with } \psi=0 \text { on all boundaries })
$$

is used.

The above equations let appears some dimensionless parameters that govern the problem, namely, the solid volume fraction $\Phi$, the aspect ratio of the enclosure, $A$, the Peclet, $P e$, Reynolds, $R e$, and Richardson, $R i$, numbers. For the last four, the expressions are

$$
A=\frac{L^{\prime}}{H^{\prime}}, P e=\frac{U_{0}^{\prime} H^{\prime}}{\alpha_{f}}, R e=\frac{U_{0}^{\prime} H^{\prime}}{v_{f}} \text { and } R i=\frac{g \beta_{f} q^{\prime} H^{\prime 2}}{k_{f} U_{0}^{\prime 2}}
$$

Note that

$$
P e=P r R e \text { and } R i=\frac{G r}{R e^{2}}=\frac{R a}{P e R e}
$$

where

$$
G r=\frac{g \beta_{f}^{\prime} q^{\prime} H^{\prime 4}}{v_{f}^{2} k_{f}}, \operatorname{Pr}=\frac{v_{f}}{\alpha_{f}} \text { and } \operatorname{Ra}=\operatorname{Pr} G r
$$

are the Grashof, Prandtl and Rayleigh numbers, respectively.

The local heat transfer, through the nanofluid-filled cavity, can be expressed in terms of the local Nusselt number defined as

$$
N u(y)=\frac{h L^{\prime}}{k_{f}}=\frac{q^{\prime}}{\Delta T^{\prime}} \frac{L^{\prime}}{k_{f}}=\frac{L^{\prime}}{H^{\prime}} \frac{\Delta T^{*}}{\Delta T^{\prime}}=\frac{A}{\Delta T}=\frac{1}{\Delta T / A}
$$

where $h$ is the heat exchange coefficient, $\Delta T^{*}=q^{\prime} H^{\prime} / k_{f}$ a characteristic temperature and $\Delta T=T(0, y)-T(A, y)$ the side to side dimensionless local temperature difference. This definition is based on the thermal conductivity of the base fluid, $k_{f}$, which seems logical since, according to Corcione [3], $N u$ that would describe the thermal performance of the enclosure, with immediacy, should vary in the same manner as $h$ and vice versa. However, Equation (25) is notoriously inaccurate owing to the uncertainty of the temperature values evaluated at the two vertical walls (edge effects). Instead, $N u$ is calculated on the basis of a temperature difference between two vertical sections, far from the end sides, as suggested by Lamsaadi et al. [19]. Thus, by analogy with Equation (25), and considering two infinitesimally close sections, $N u$ can be expressed by

$$
N u(y)=\lim _{\delta x \rightarrow 0} \frac{\delta x}{\delta T}=\lim _{\delta x \rightarrow 0} \frac{1}{(\delta T / \delta x)}=-\frac{1}{(\partial T / \partial x)_{x=A / 2}}
$$


where $\delta x$ is the distance between two symmetrical sections with respect to the central one.

The corresponding average Nusselt number is calculated, at different locations, from

$$
\overline{N u}=\int_{0}^{1} N u(y) \mathrm{d} y
$$

\section{Numerics}

Equations (14)-(17) associated with Equations (18)-(20) have been solved by using a finite volume method and SIMPLER algorithm in a staggered uniform grid system [20]. A second order back-wards finite difference scheme has been employed to discretise the temporal terms appearing in Equations (15)-(17). A line-by-line tridiagonal matrix algorithm with relaxation has been used in conjunction with iterations to solve the nonlinear discretised equations. The convergence has been considered as reached when $\sum_{i, j}\left|f_{i, j}^{k+1}-f_{i, j}^{k}\right|<10^{-5} \sum_{i, j}\left|f_{i, j}^{k+1}\right|$, where $f_{i, j}^{k}$ stands for the value of $u, v, p$ or $T$ at the $k^{\text {th }}$ iteration level and grid location $(i, j)$ in the plane $(x, y)$. The mesh size has been chosen so that a best compromise between running time and accuracy of the results may be found. The procedure has been based on grid refinement until the numerical results agree, within reasonable accuracy, with the analytical ones, obtained from the parallel flow approach developed in the next section. Hence, as shown in Table 1, a uniform grid size of $160 \times 40$ has been selected for $A=8$ (value used for the numerical computations) and has been estimated sufficient to model accurately the flow and temperature fields within the cavity. The time step size, $\delta t$, has been varied in the range $10^{-7} \leq \delta t \leq 10^{-4}$, depending on the values of the governing parameters.

\section{Approximate Parallel Flow Analytical Solution}

As can be seen from Figures 2-4, displaying streamlines (left) and isotherms (right), the flow and temperature fields exhibit a parallel aspect and a linear stratification, respectively, in the most part of the cavity, for $A=8$ and various values of $R e, R i$ and $\Phi$. Accordingly, the following simplifications

$$
\begin{aligned}
& u(x, y)=u(y), v(x, y)=0, \psi(x, y)=\psi(y) \\
& \text { and } T(x, y)=C(x-A / 2)+\theta(y)
\end{aligned}
$$

where $C$ is unknown constant temperature gradient in the $x$-direction, are possible, which leads to the ordinary nondimensional governing equations:

$$
\frac{\mathrm{d}^{3} u}{\mathrm{~d} y^{3}}=\bar{\alpha} \Omega \operatorname{Re} R i \frac{\partial T}{\partial x}=\bar{\alpha} \Omega \operatorname{Re} R i C
$$

\begin{tabular}{|c|c|c|c|c|c|c|}
\hline \multirow{2}{*}{$\begin{array}{l}\text { Grids } \\
\Phi\end{array}$} & \multicolumn{6}{|c|}{$(160 \times 20)$} \\
\hline & & & $\psi_{c}$ & $\overline{N u}$ & & \\
\hline 0.0 & & & 0.4429 & 4.7200 & & \\
\hline 0.1 & & & 0.4179 & 3.6429 & & \\
\hline 0.2 & & & 0.3533 & 2.9500 & & \\
\hline Grids & \multicolumn{2}{|c|}{$(120 \times 40)$} & \multicolumn{2}{|c|}{$(160 \times 40)$} & \multicolumn{2}{|c|}{$(200 \times 400)$} \\
\hline$\Phi$ & $\psi_{c}$ & $\overline{N u}$ & $\psi_{c}$ & $\overline{N u}$ & $\psi_{c}$ & $\overline{N u}$ \\
\hline 0.0 & 0.4427 & 4.6446 & 0.4427 & 4.6444 & 0.4428 & 4.6440 \\
\hline 0.1 & 0.4169 & 3.5948 & 0.4171 & 3.5952 & 0.4171 & 3.5949 \\
\hline 0.2 & 0.3504 & 2.9018 & 0.3504 & 2.9024 & 0.3506 & 2.9018 \\
\hline Grids & \multicolumn{6}{|c|}{$(160 \times 60)$} \\
\hline$\Phi$ & & & $\psi_{c}$ & $\overline{N u}$ & & \\
\hline 0.0 & & & 0.4427 & 4.6444 & & \\
\hline 0.1 & & & 0.4179 & 3.5952 & & \\
\hline 0.2 & & & 0.3502 & 2.9024 & & \\
\hline
\end{tabular}

Table 1. Accuracy tests conducted with $R e=1, R i=10^{3}$ and various values of $\Phi$.

$$
\frac{\bar{\alpha}}{P e} \frac{\mathrm{d}^{2} \theta}{\mathrm{d} y^{2}}=C u
$$

with

$$
\begin{aligned}
u=\frac{\mathrm{d} \theta}{\mathrm{d} y}=0 \text { for } y= & 0 \text { and } u+1=\frac{\mathrm{d} \theta}{\mathrm{d} y}=0 \text { for } y=1 \\
& \int_{0}^{1} u(y) \mathrm{d} y=0 \\
& \int_{0}^{1} \theta(y) \mathrm{d} y=0
\end{aligned}
$$

as boundary, return flow and mean temperature conditions, respectively.

Using such an approach, the solution of Equations (29) and (30), satisfying Equations (31)-(33), is

$$
\begin{gathered}
u(y)=\frac{\bar{\alpha}}{12} \Omega \operatorname{ReRiC}\left(2 y^{3}-3 y^{2}+y\right)+\left(-3 y^{2}+2 y\right) \\
\theta(y)=\frac{1}{12} \Omega \operatorname{Ra} C^{2}\left(\frac{y^{5}}{10}-\frac{y^{4}}{4}+\frac{y^{3}}{6}-\frac{1}{120}\right) \\
+\frac{P e C}{\bar{\alpha}}\left(-\frac{y^{4}}{4}+\frac{y^{3}}{3}-\frac{1}{30}\right)
\end{gathered}
$$

The expression of the stream function, $\psi(y)$, can be deduced by integration of Equation (21), taking into account of the corresponding boundary conditions and Equation (34), which gives: 


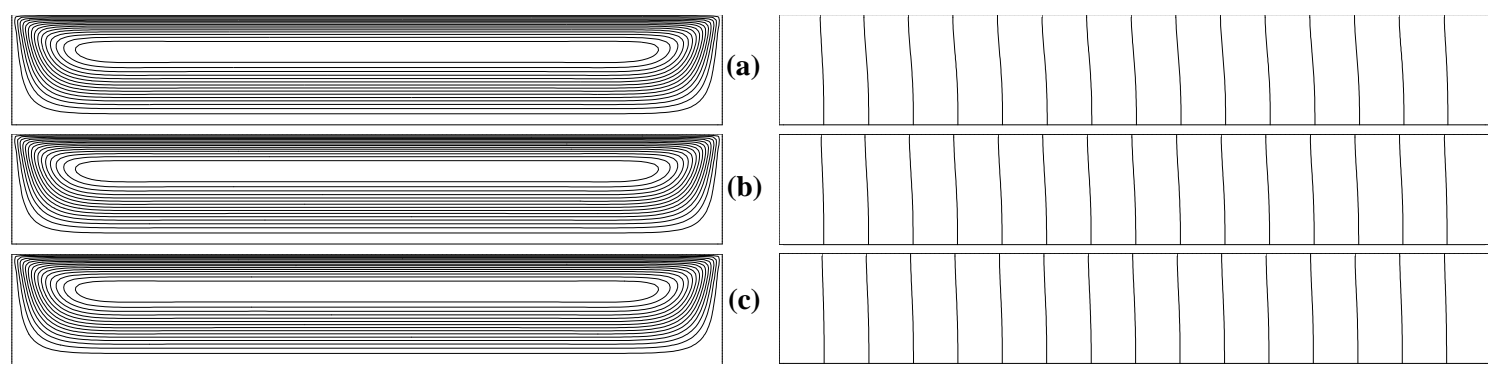

(1)

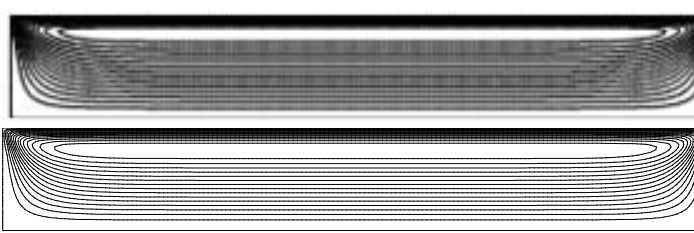

(a)

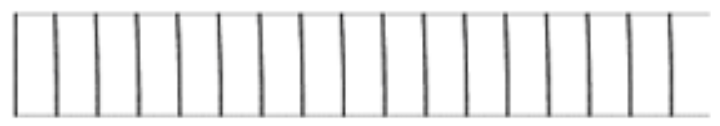

(b)

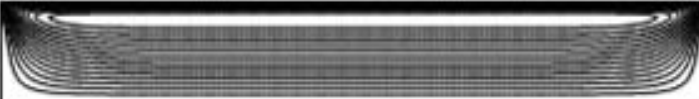

(c)
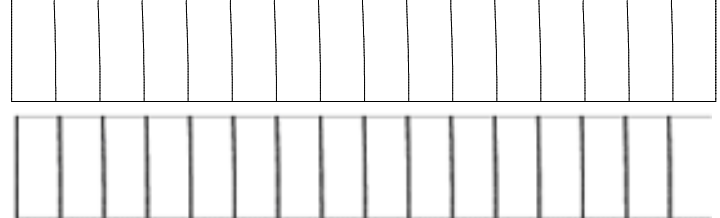

(2)

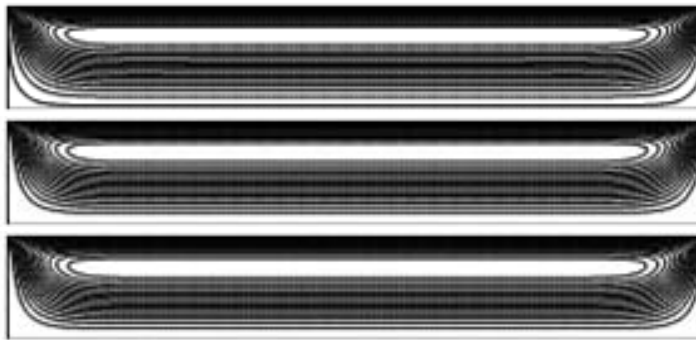

(a)

(b)

(c)
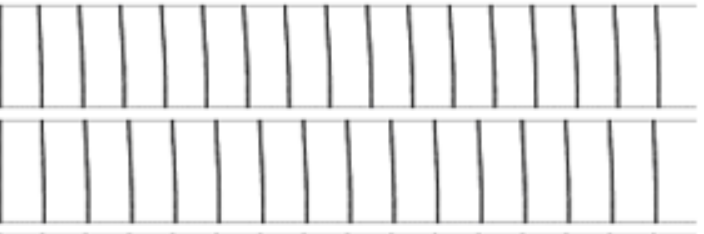

(3)

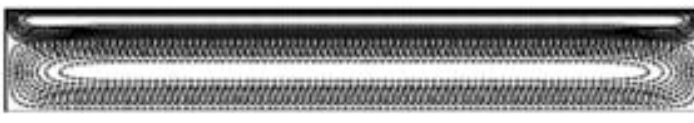

(a)
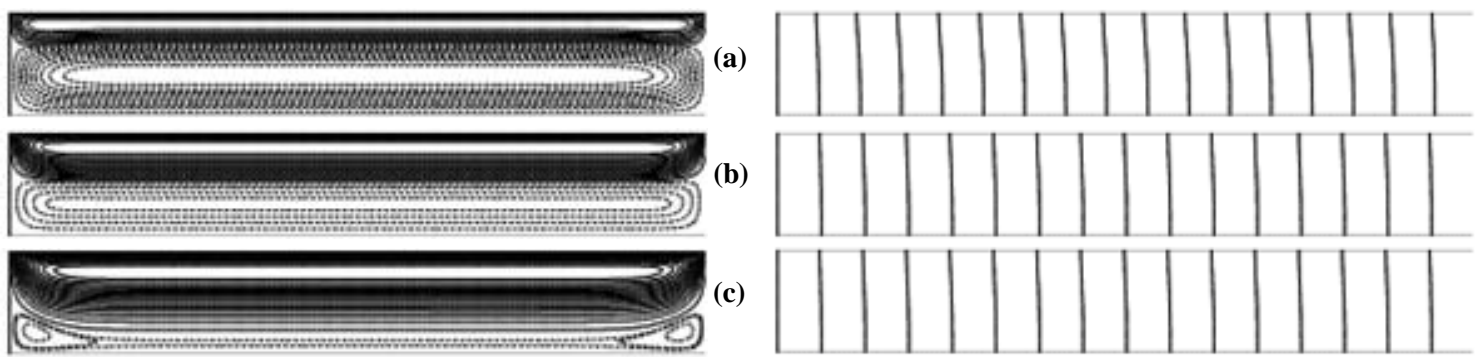

(b)

(c)

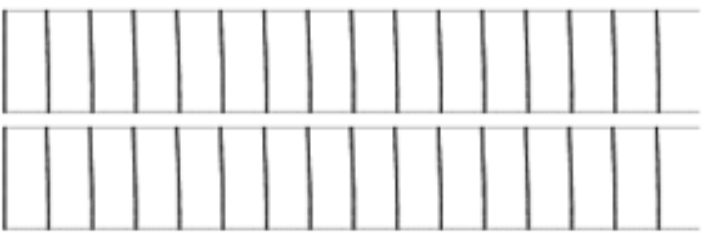

(4)

Figure 2. Streamlines (left) and isotherms (right) for $R e=0.1$ and various values of $\Phi$ ((a) $\Phi=0.1$, (b) $\Phi=0.1$ and (c) $\Phi=0.2)$ and $R i\left((1) R i=1,(2) R i=10\right.$, (3) $R i=10^{2}$ and (4) $R i=10^{3}$ ).

$$
\psi(y)=\frac{\bar{\alpha}}{12} \Omega \operatorname{ReRiC}\left(\frac{y^{4}}{2}-y^{3}+\frac{y^{2}}{2}\right)+\left(-y^{3}+y^{2}\right)
$$

where $\Omega=\frac{\bar{\beta}}{\bar{\rho} \bar{\alpha} \bar{\nu}}$. Therefore, the flow intensity is

$$
\psi_{c}=\operatorname{Sup}\left(\psi_{\max },\left|\psi_{\min }\right|\right)
$$

where $\psi_{\max }$ and $\left|\psi_{\min }\right|$ are the extrema of $\psi(y)$ at the central vertical section of the enclosure $(x=A / 2)$. They correspond to the intensities of forced and natural convections, respectively.
On the other hand, according to Bejan [21], the energy balance in $x$-direction is

$$
\int_{0}^{1}-\frac{\partial T}{\partial x} \mathrm{~d} y+\frac{P e}{\bar{\alpha}} \int_{0}^{1} u T \mathrm{~d} y=\int_{0}^{1}-\left(\frac{\partial T}{\partial x}\right)_{x=0 \text { or } A} \mathrm{~d} y
$$

In particular, in the parallel flow region and with the application of Equation (18), Equation (38) becomes:

$$
-C+\frac{P e}{\bar{\alpha}} \int_{0}^{1} u \theta \mathrm{d} y=\frac{1}{\bar{k}}
$$

which, when substituted to Equations (34) and (35), gives the following transcendental equation: 


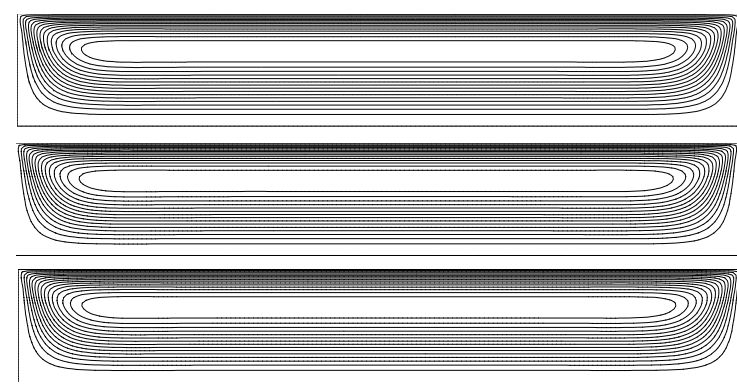

(a)

(b)

(c)

(1)
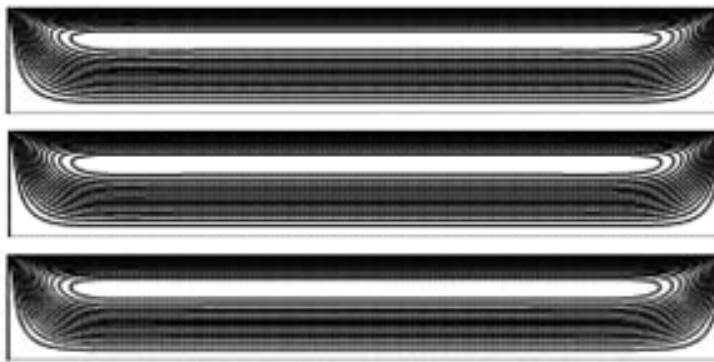

(a)

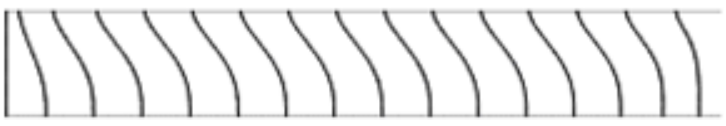

(b)

(c)

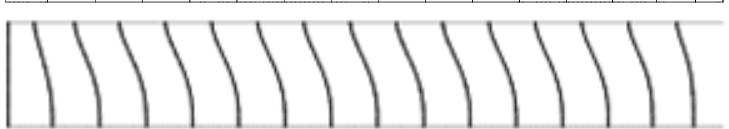

(2)
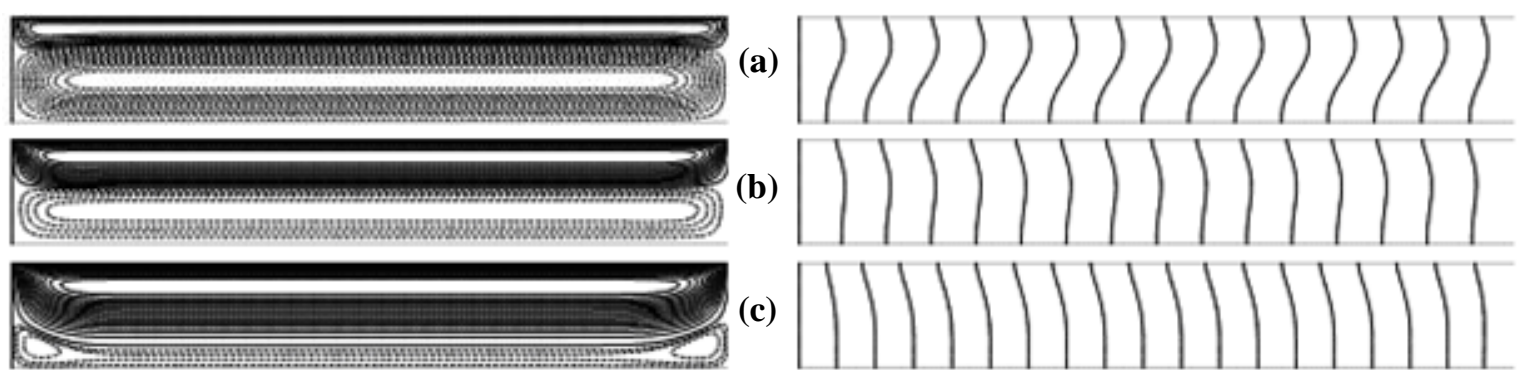

(3)

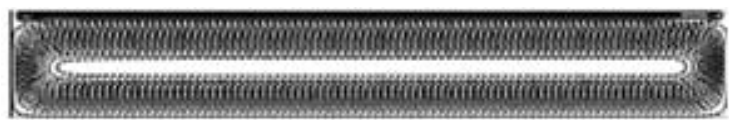

(a)

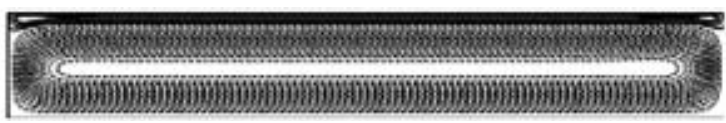

(b)
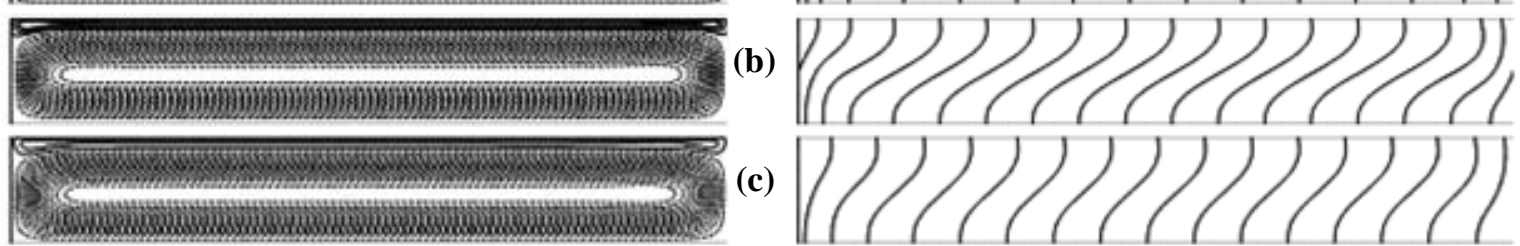

(c)

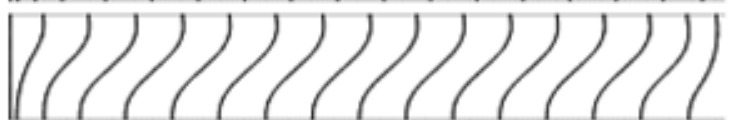

(4)

Figure 3. Streamlines (left) and isotherms (right) for $R e=1$ and various values of $\Phi$ ((a) $\Phi=0.1$, (b) $\Phi=0.1$ and (c) $\Phi=0.2$ ) and $R i\left((1) R i=1,(2) R i=10\right.$, (3) $R i=10^{2}$ and (4) $R i=10^{3}$ ).

$$
\frac{1}{\bar{k}}+\left(1+\frac{P e^{2}}{105 \bar{\alpha}^{2}}\right) C+\frac{\Omega P e R a}{3360 \bar{\alpha}} C^{2}+\frac{\Omega^{2} R a^{2}}{362,880} C^{3}=0
$$

whose solution, via Newton-Raphson method, for given $P e, R a$ and $\Phi$, leads to $C$.

Finally, taking into account of Equations (26) and (27), the Nusselt number is constant and can be expressed as

$$
N u=\overline{N u}=-\frac{1}{C}
$$

\section{Results and Discussion}

With boundary conditions of Neumann kind (uniform heat flux imposed to vertical walls) the flow and thermal fields, and thermo-convective characteristics become parallel, stratified and independent on the enclosure aspect ratio, $A$, respectively, when this parameter tends to be large enough. In our situation, this has been occurred with $A=8$ in the limit of the explored values of $R e$ $(0.1 \leq R e \leq 10), R i \quad\left(1 \leq R i \leq 10^{3}\right), \Phi \quad(0 \leq \Phi \leq 0.2)$, and $\operatorname{Pr}=7$ (water based mixtures). Consequently, the 


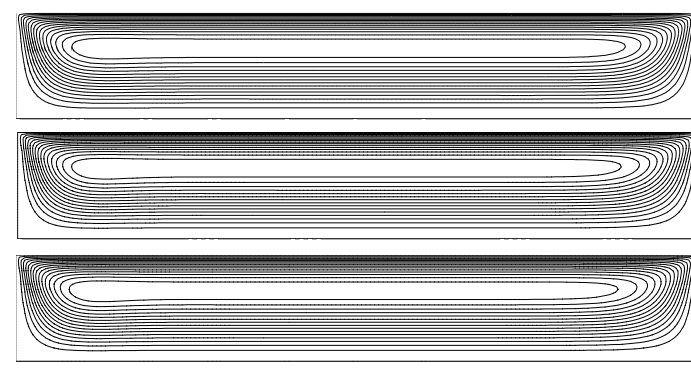

(c)

(1)

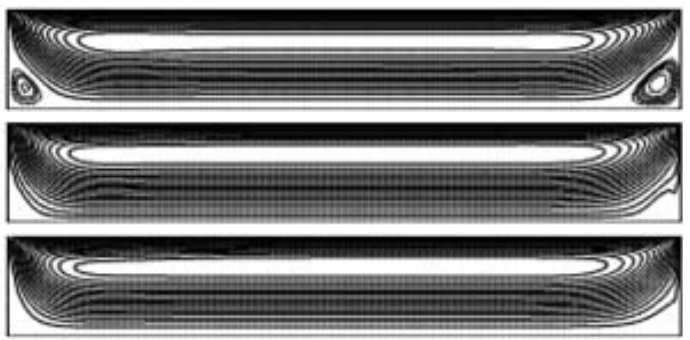

(a)

(b)

(c)
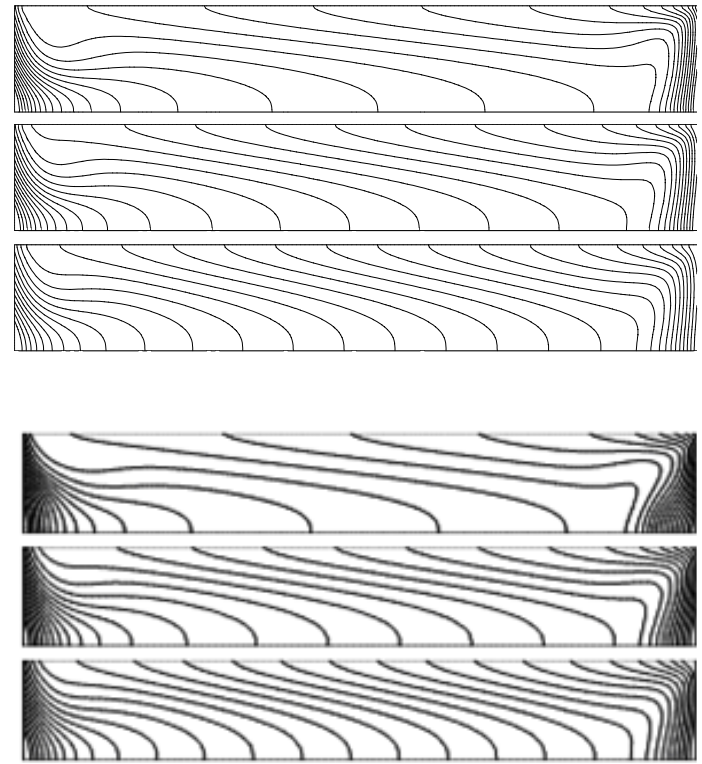

(2)

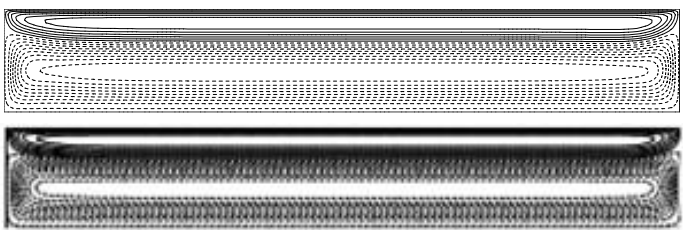

(a)

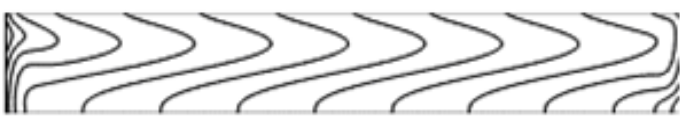

(b)

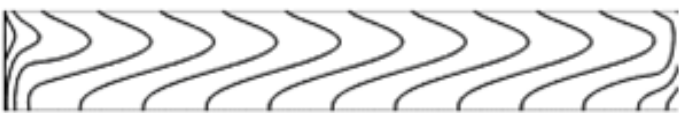

(c)

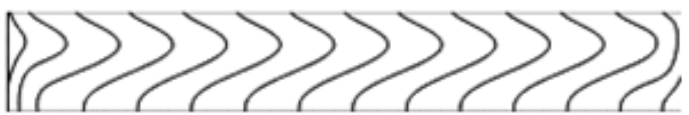

(3)

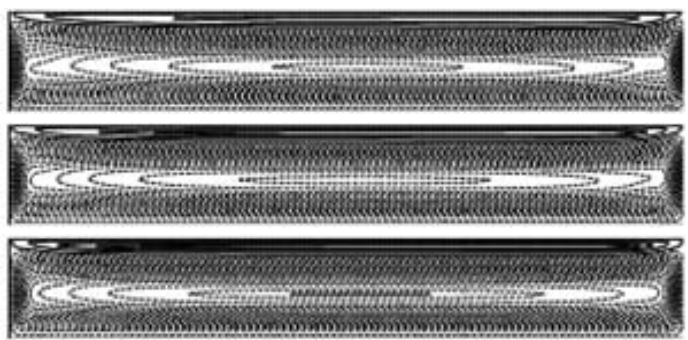

(a)

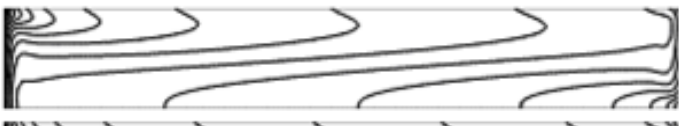

(b)

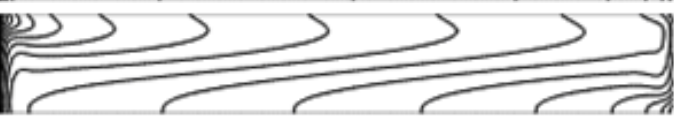

(c)

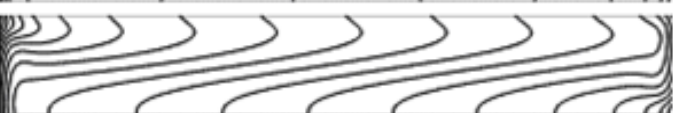

(4)

Figure 4. Streamlines (left) and isotherms (right) for $\operatorname{Re}=10$ and various values of $\Phi((\mathrm{a}) \Phi=0.1$, (b) $\Phi=0.1$ and (c) $\Phi=0.2)$ and $R i\left((1) R i=1,(2) R i=10,(3) R i=10^{2}\right.$ and (4) $R i=10^{3}$ ).

mixed convection flow developed within the enclosure is governed only by four dimensionless parameters, namely, $R e, R i$, in accordance with Equations (22)-(24), $\Phi$ and possibly the type of nanoparticles, even if the present study is limited to $\mathrm{Cu}$-water nanofluid, with the thermophysical properties of $\mathrm{Cu}$ and water given in Table 2 [22]. The effects of these parameters on the flow and thermal fields and the resulting heat transfer will be now discussed.

\subsection{Flow and Thermal Patterns}

Typical contours of streamlines (left) and isotherms (right) are presented in Figures 2-4 for each $R e$ and various $R i$ and $\Phi$. First of all, remember that, except in the end sides, the flow is parallel to the horizontal boundaries and the temperature is linearly stratified in the horizontal direction. On the other hand, as can be seen from Figure 2 corresponding to $R e=0.1$, the shear effect due to moving top wall is dominant for $R i$ small $(=1)$, relatively small (= $10)$ and moderate $\left(=10^{2}\right)$, since the flow is unicellular and counterclockwise with streamlines crowded near this boundary causing the lost of their own symmetry with respect to the horizontal mid-plane and this, whatever the value of $\Phi$. In contrast, for relatively high $R i\left(=10^{3}\right)$, a 
clockwise cell, whose size reduces with an increasing $\Phi$, is generated by buoyancy effect in the lower part of the cavity, i.e. under that due to lid-driven effect. The influence of $\Phi$ can be explained by the fact that an increase of this parameter leads to an increase of the effective viscosity, which, in its turn, tends to decelerate the buoyancy flow and accelerate the shear one. In fact, the viscosity is a manifestation of surface phenomena and therefore can only favour the shear flow. As for the isotherms, they remain almost vertical synonymous of pseudo-conduction regime (i.e. low convection regime, $R e=0.1$ ). An increase of $R e$ to 1 (Figure 3) anticipates the buoyancy flow, since the related cell takes place at $R i=10^{2}$, with a decreasing size with $\Phi$ like before (i.e. $R e=0.1$ ). However, such a cell tends to be large when passing to $R i$ $=10^{3}$, which is normal if one refer to Equation (22). In such a situation, the isotherms, which are tilted, with respect to $y$-axis, in the counterclockwise direction (i.e. that of the shear flow), gradually change direction of tilting as the buoyancy flow takes importance (i.e. while increasing $R i$ ). But, with the presence of nanoparticles (i.e. with increasing $\Phi)$, such a change seems to occur slowly, depending on $R i$. Finally, for $R e=10$ (Figure 4), two small clockwise eddies develop in the bottom left and right corners of the enclosure, for $R i=10$ (Figure 4(2a)), as a result of buoyancy driven effect, although these structures may disappear wile introducing nanoparticles in the base fluid. As before, a progressive increase of $R i$ to $10^{2}$ (Figure 4(3)) makes stronger buoyancy effect and the two tiny eddies, observed previously, join each other and grow to give rise to a large buoyancy cell, whose size reduces slightly with $\Phi$, for the reasons given above. A further increase of $R i$ to $10^{3}$ (Figure 4(4)) makes bigger enough buoyancy cell so that the effect of $\Phi$, on this, cannot be detected, which is obvious since, according to Equation (22), inertia effects due to gravity prevail over those associated with top moving wall. At the same time, the isotherms appear to be nearly tilted in the direction of the strongest flow (shear flow for $R i=1$ and 10, and buoyancy flow for $R i=10^{3}$ ). Between the two case, i.e. for $R i=10^{2}$, both the tendencies exist and the shape of the isotherms is strongly distorted. Here also, it is easy to see that an increase of $\Phi$ does not affect strongly the shape of isotherms.

In addition to that, the similarity notion is not respected in the present problem since a change of Re may cause big changes in flow and thermal fields for given $R i$

Table 2. Thermophysical properties of base fluid $\left(\mathrm{H}_{2} \mathrm{O}\right)$ and nanoparticles $(\mathrm{Cu})$ [22].

\begin{tabular}{ccccc}
\hline & $\rho\left(\mathrm{kg} / \mathrm{m}^{3}\right)$ & $C_{P}(\mathrm{~J} / \mathrm{kg} \cdot \mathrm{k})$ & $k(\mathrm{~W} / \mathrm{m} \cdot \mathrm{k})$ & $\beta \times 10^{5}(1 / \mathrm{k})$ \\
\hline $\mathrm{H}_{2} \mathrm{O}$ & 997.1 & 4179 & 0.613 & 21 \\
$\mathrm{Cu}$ & 8933 & 385 & 401 & 1.67 \\
\hline
\end{tabular}

and $\Phi$ (compare, for example, Figures 2(3a)-4(3a) corresponding to $R i=10^{2}$ and $\left.\Phi=0\right)$. Also, for the case $R i=$ 1 , although buoyancy and lid-driven effects have the same magnitude, the shear flow is dominant because of the small depth of the enclosure (shallow cavity).

\subsection{Validation of the Approximate Parallel Flow Analytical Solution}

In order to validate the approximate analytical solution, the numerical results (full circles) are compared to those obtained analytically (solid lines), as displayed in Figures 5-7 giving stream function (left) and temperature (right) profiles along the $y$-axis at the mid-length of the cavity, $\psi(A / 2, y)$ and $T(A / 2, y)$, respectively. As can be seen, the agreement between the two types of results is quite perfect, which confirms the existence of an analytical solution and validates mutually the parallel flow hypothesis and the elaborated computing code.

Moreover, computed and calculated values of the stream function at the vertical central section of the cavity, $\psi_{c}$, and mean Nusselt number, $\overline{N u}$, presented in Figures 8-10, show also good agreement between the analytical and numerical results for a wide range of $R i$, and various values of $R e$ and $\Phi$.

\subsection{Stream Function and Temperature Distributions along the Vertical Central Section}

Although the results (Figures 5-7) of subsection 5.2 are related to the core region, where the parallel flow concept is valid, they confirm mostly those discussed in subsection 5.1 and demonstrate that, in general, an increase of $\Phi$ amplifies and reduces the strengths of forced and natural flows, respectively, except in some cases where $\Phi$ remains almost without influence due to mixing effects that occur at $R e=10$ (Figure 7).

The presence of a maximum in the stream function profile indicates that the flow is unicellular counterclockwise, driven mainly by the moving top wall. When this profile presents, simultaneously, two extrema, maximum and minimum, this means that both the shear and buoyancy driven flows coexist and the flow regime is bicellular. The maximum (positive extremum) corresponds to shear flow, which is counterclockwise, and the minimum (negative extremum) is related to buoyancy flow that is clockwise.

Generally, the temperature profile presents two or three zones with positive and negative signs, namely $(+,-),(-,+,-)$ and $(-,+)$, depending on the flow nature and the competition between lid and buoyancy driven effects. Thus, with a dominant lid-driven effect, the shear flow warms the bottom $(y=0)$ by transporting the heat from the left hot side and cools the top $(y=1)$ after pass- 

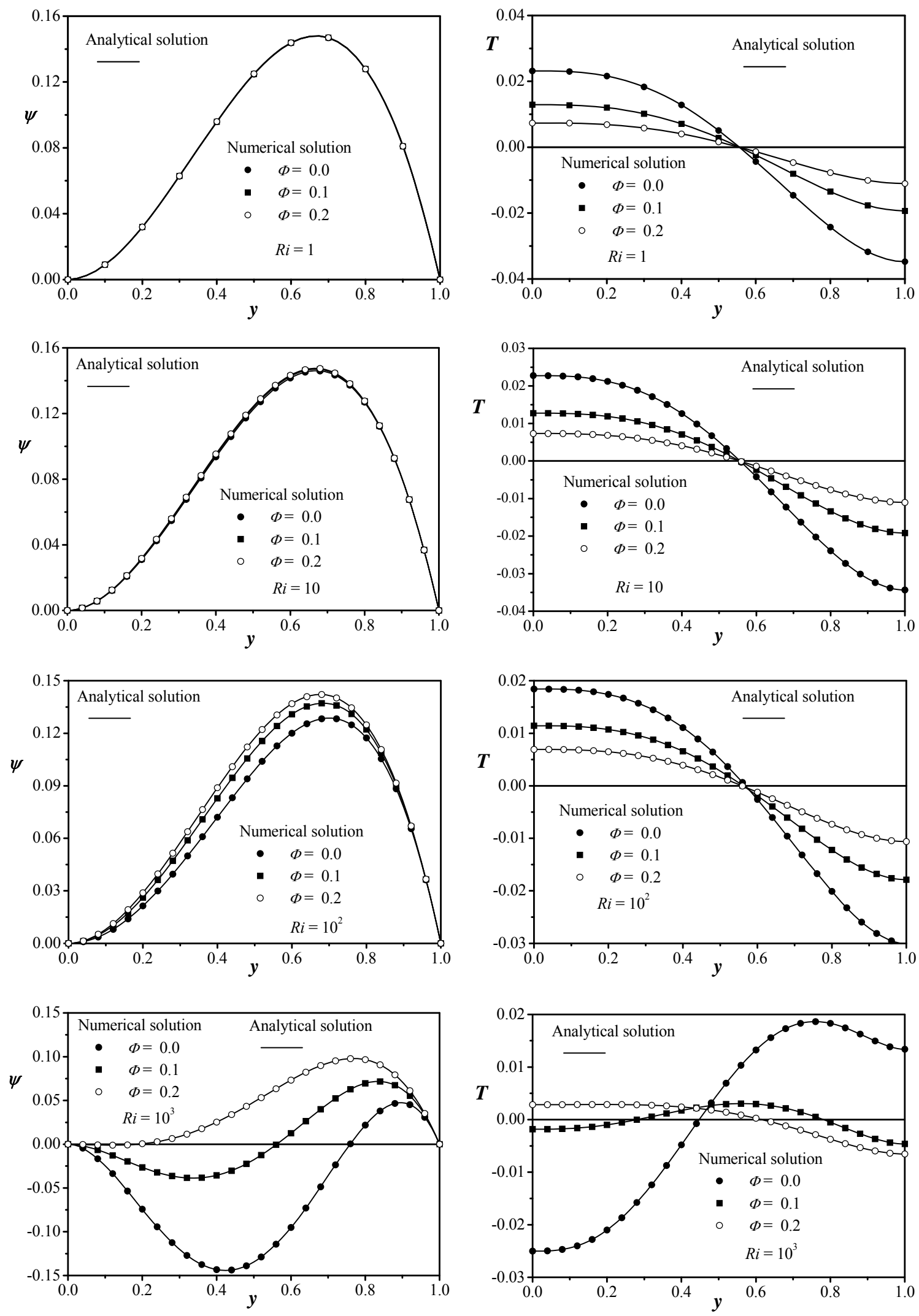

Figure 5. The stream function (left) and temperature (right) profiles at mid-length of the cavity, along the vertical coordinate, for $R e=0.1$ and various values of $\Phi$ and $R i$. 

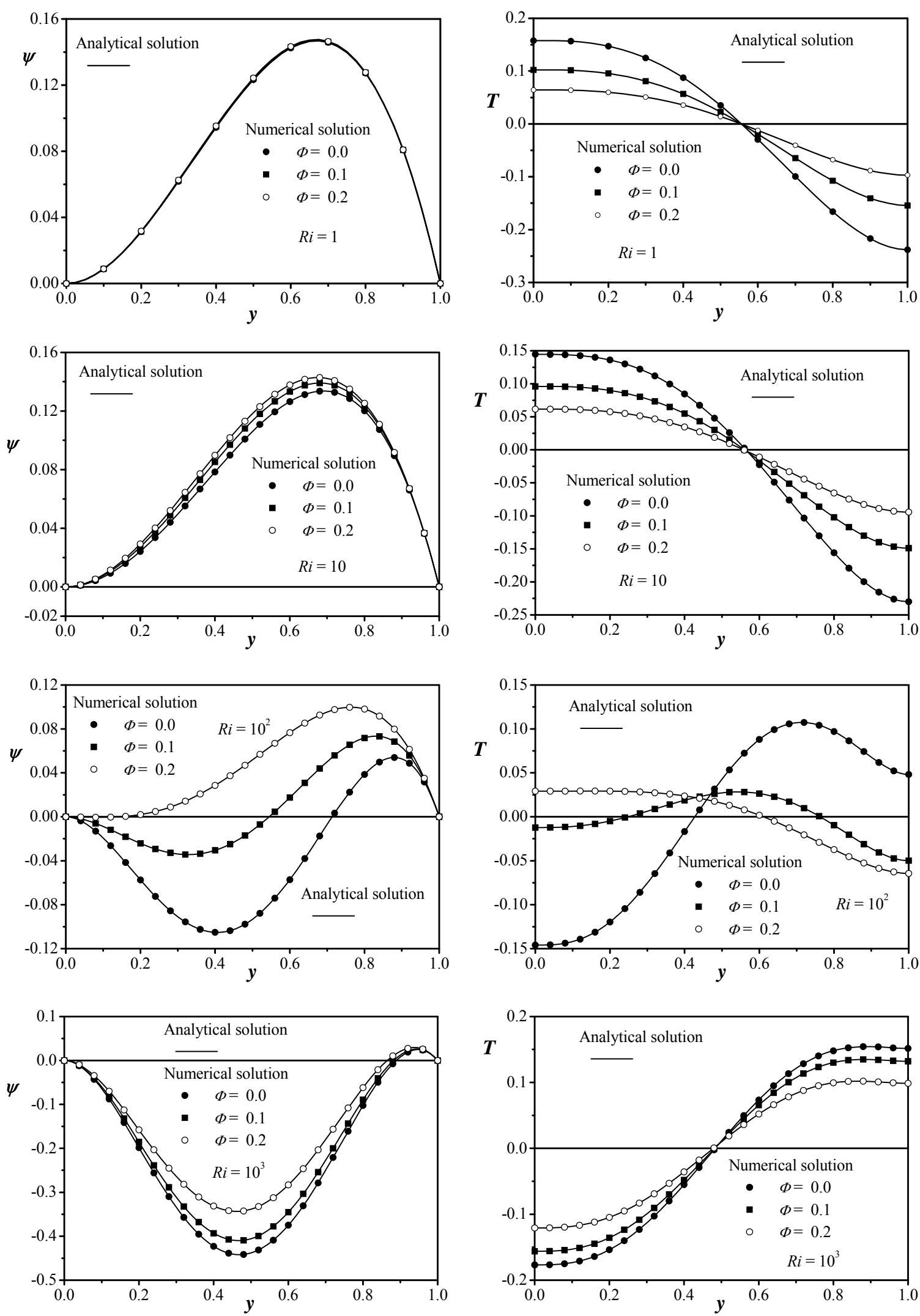

Figure 6. The stream function (left) and temperature (right) profiles at mid-length of the cavity, along the vertical coordinate, for $R e=1$ and various values of $\Phi$ and $R i$. 

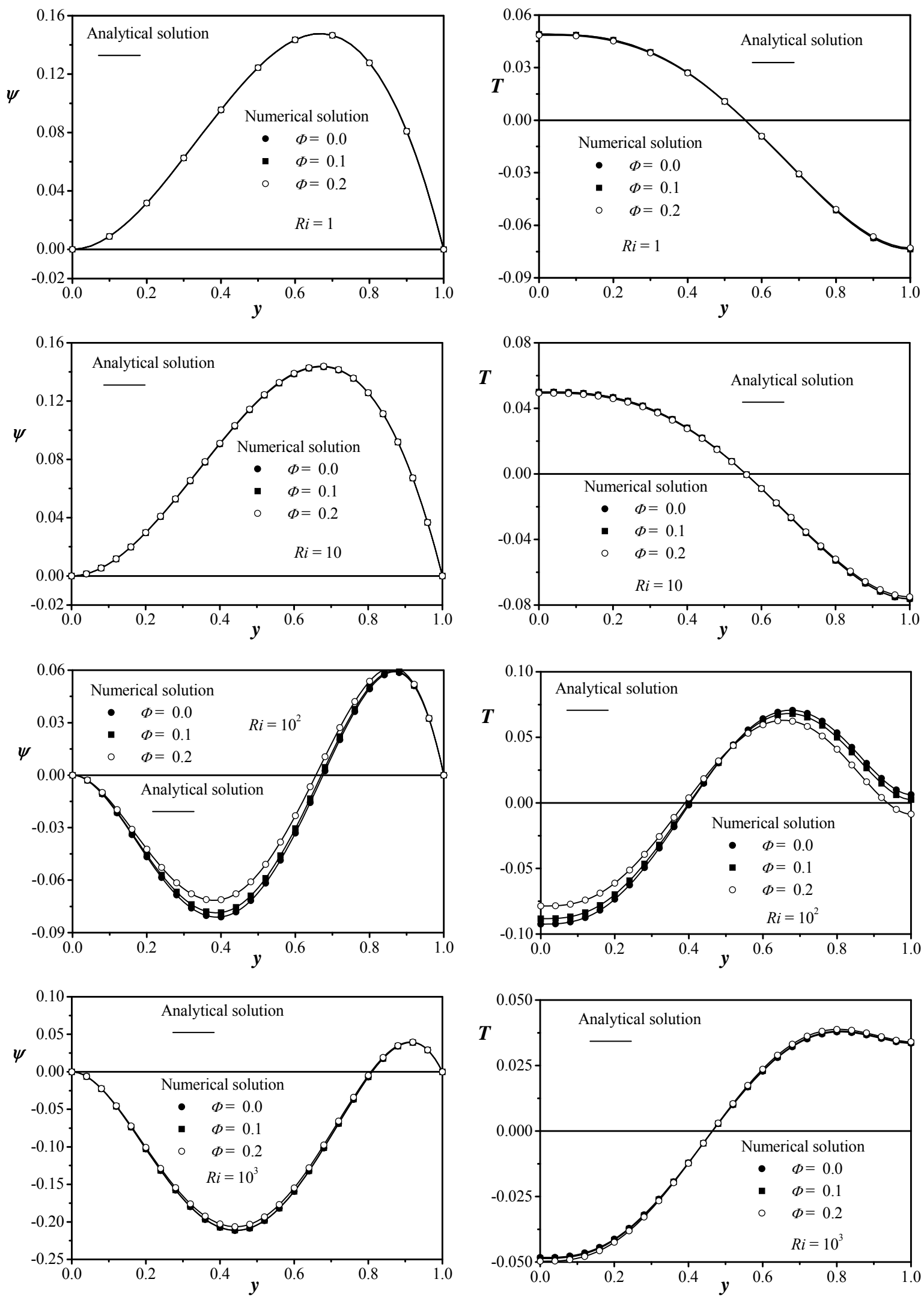

Figure 7. The stream function (left) and temperature (right) profiles at mid-length of the cavity, along the vertical coordinate, for $R e=10$ and various values of $\Phi$ and $R i$. 

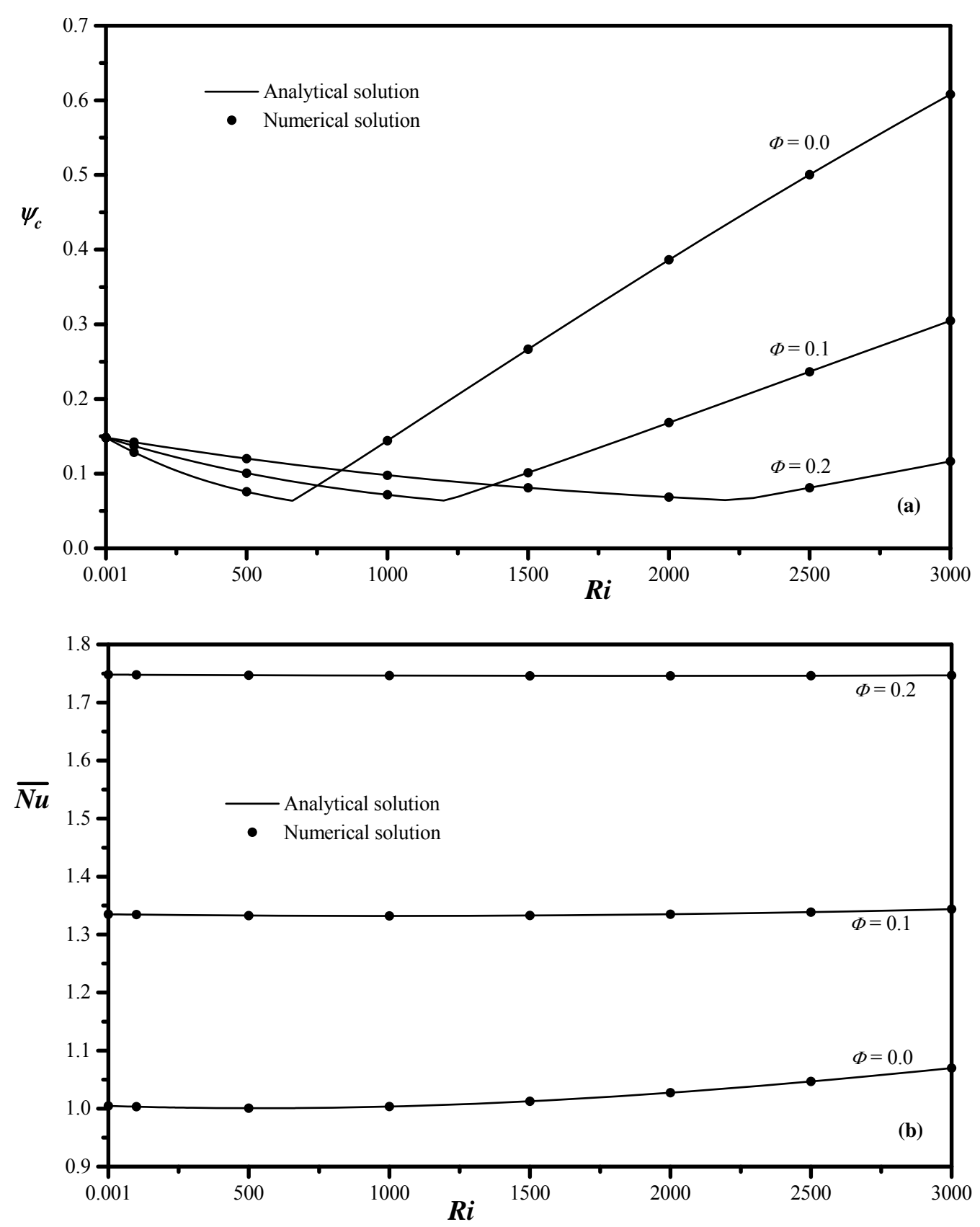

Figure 8. Flow intensity (a) and heat transfer rate (b) versus $R i$, for $R e=0.1$ and various values of $\Phi$.

ing near the right cold side. This is the case of a temperature profile of signs $(+,-)$. With competing shear and buoyancy flows, the temperature sign is such that the corresponding counterclockwise and clockwise cells act so that the interface between them is warm, the bottom is cold and the top is warm $(-,+)$ or cold $(-,+,-)$ depending on whether shear or buoyancy effect is dominant, and on $\Phi$.

\subsection{Flow Intensity and Heat Transfer Rate}

For further analysis of the problem, the flow intensity (top), $\psi_{c}$, and heat transfer rate (bottom), $\overline{N u}$, are reported, against $R i$, in Figures 8-10, for each $R e$ and various $\Phi$.

It is easy to observe that $\psi_{c}$ exhibits in general two tendencies, whose expanse depends on $R e$ and $\Phi$. The first one is characterized by a decrease of $\psi_{c}$ with $R i$, expressing a reduction of the strength of the shear flow until a minimum reached at particular value of $R i$, which increases and decreases with $\Phi$ and $R e$, respectively. The second one corresponds to an increase of $\psi_{c}$ with $R i$, beyond the minimum observed, and expresses an aug- 

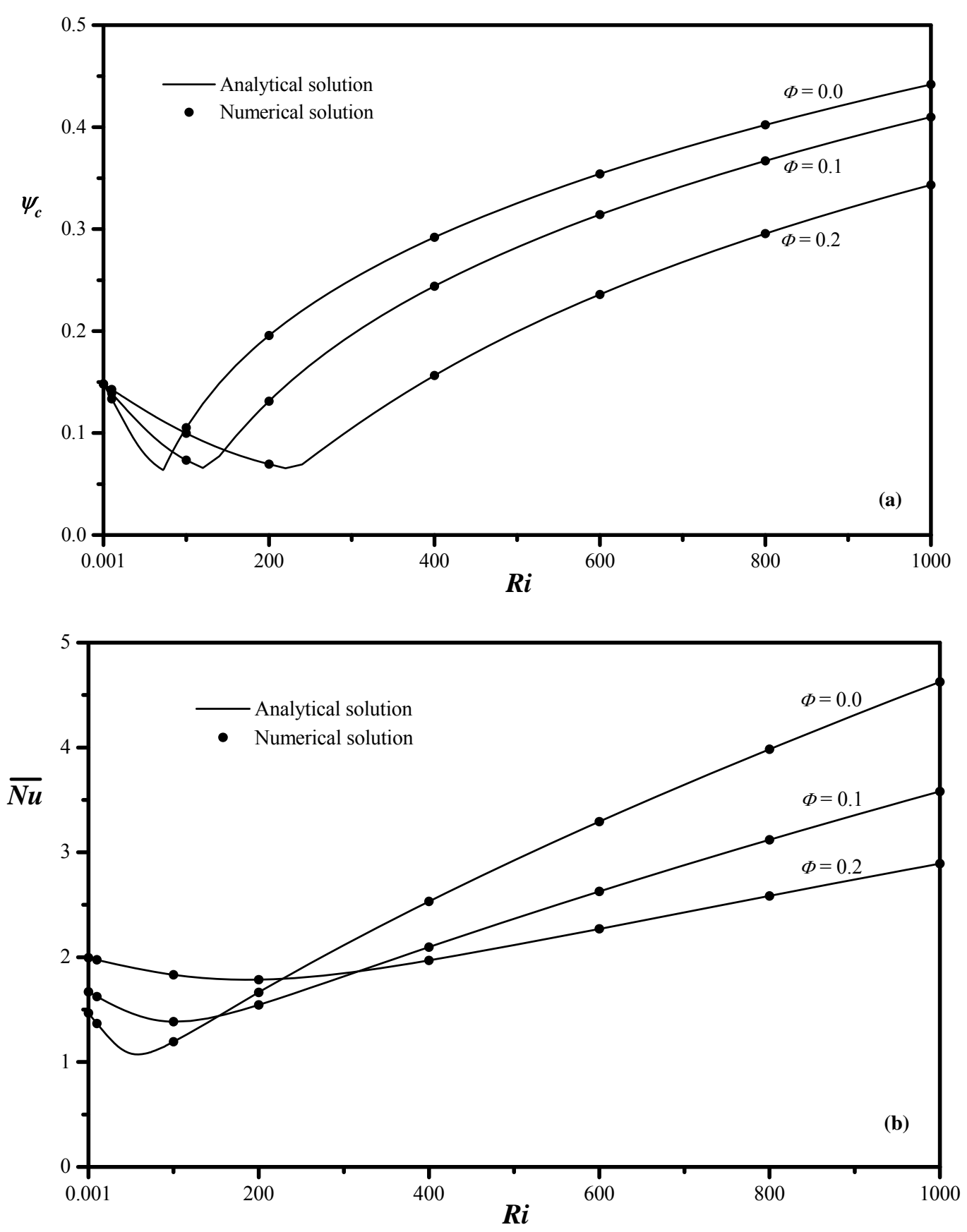

Figure 9. Flow intensity (a) and heat transfer rate (b) versus $R i$, for $R e=1$ and various values of $\Phi$.

mentation of the strength of the buoyancy flow which reduces with $\Phi$. It can be seen, also, that an increase of $R e$ leads, first, to an increase of $\psi_{c}$ and, second, to a decrease of this quantity (compare Figures 8-10, obtained for $R e=0.1,1$ and 10 , respectively). This is due, probably, to a change of the dominant role from one regime to another.

With regard to $\overline{N u}$, a slight increase of this quantity, with $R i$, is observed for $\Phi=0$, but such a tendency disappears, as $\Phi$ increases, leading to a constant $\overline{N u}$. This behaviour is the consequence of an increase of the effective viscosity with $\Phi$, which acts to slow down the motion, particularly, for $R e=0.1$ where mixed convection is weak (Figure 8). Moreover, for $R e=1$ and 10, two trends of evolution appear for $\overline{N u}$, as for $\psi_{c}$, since $\overline{N u}$ decreases and increases with $R i$ on both sides of a minimum depending on $\Phi$ (Figures 9 and 10). The first trend, which corresponds to a short range of $R i$, whose expanse increases with $\Phi$, is related to a dominant liddriven flow. In contrast, the second trend, which stretches 

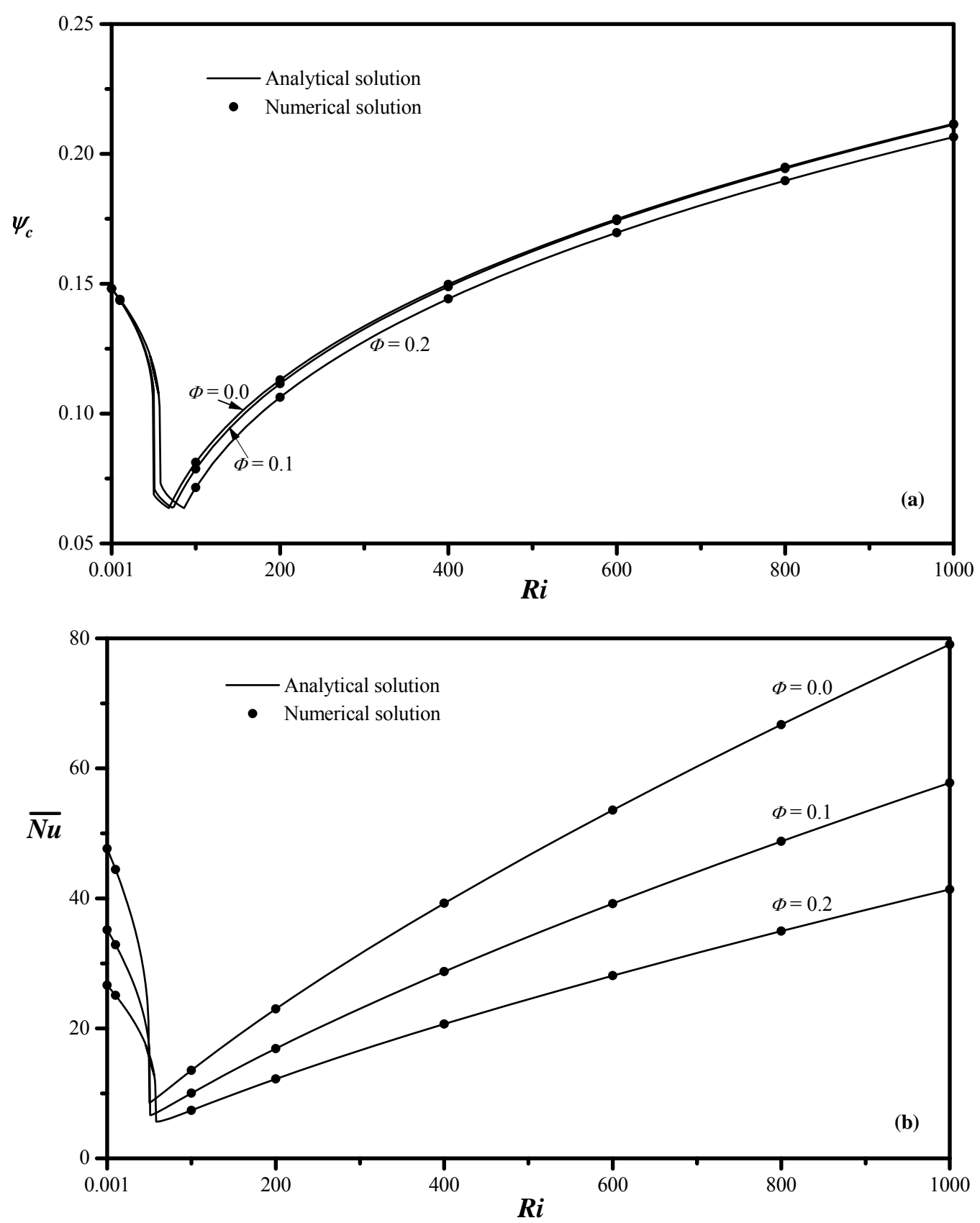

Figure 10. Flow intensity (a) and heat transfer rate (b) versus $R i$, for $R e=10$ and various values of $\Phi$.

over a large range of $R i$, is associated with a prevailing buoyancy-driven flow. Last, Figures 8-10 show also the quite obvious increase of $\overline{N u}$ with $R e$, conveying the favorable role of the lid-driven flow to heat transfer.

In order to examine the influence of $\Phi$ on flow intensity and heat transfer rate, the quantities $\psi_{c}$ (top) and $\overline{N u}$ (bottom) are plotted against $\Phi$, for various $R i$ and each $R e$, in Figures 11-13. For $R e=0.1$ (Figure 11), except for $R i=10^{3}$ where a decrease of $\psi_{c}$ with $\Phi$, due probably to the reduction of buoyancy effect by nanoparticles, is observed for $\Phi<7.5 \%, \psi_{c}$ and $\overline{N u}$ are, in general, increasing functions of $\Phi$. For $\underline{R e}=1$, Figure 12 shows different variations of $\psi_{c}$ and $\overline{N u}$, with $\Phi$, depending on $R i$. Hence, for $R i=1$ and $10, \psi_{c}$ is almost constant (very weak slop), while the increase of $\overline{N u}$ is clear, although it is not notable. For $R i=10^{2}, \psi_{c}$ presents a decrease and an increase on both sides of $\Phi=$ $6.25 \%$, whereas the increase of $\overline{N u}$ is monotonic. For $R i=10^{3}$ the tendency is reversed since $\psi_{c}$ and $\overline{N u}$ are decreasing functions of $\Phi$. At last, for $R e=10$ (Figure 13), $\psi_{c}$ seems to be quite unconcerned about any variation of $\Phi\left(\psi_{c} \approx\right.$ constant $)$, whilst $\overline{N u}$ undergoes 

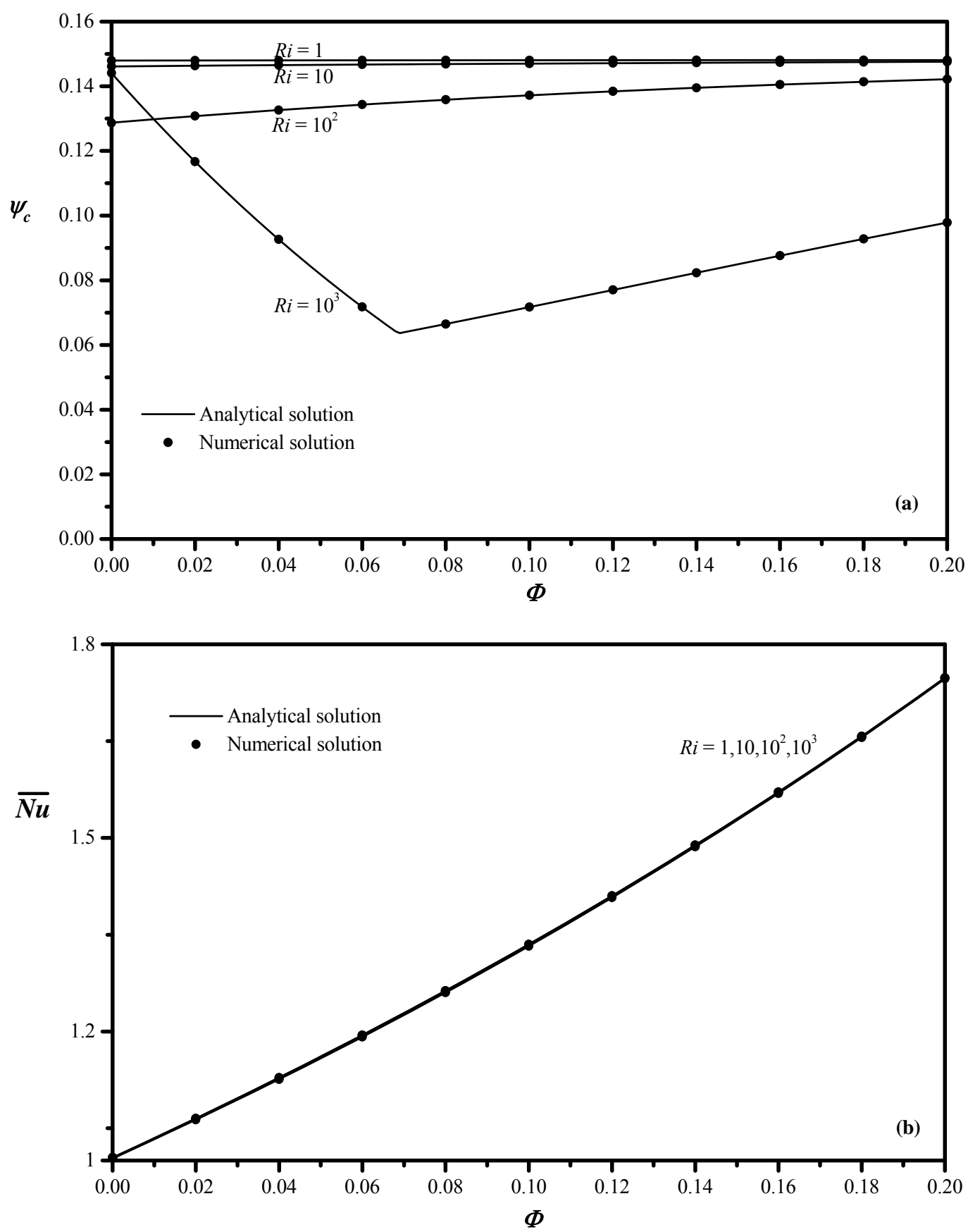

Figure 11. Flow intensity (a) and heat transfer rate (b) versus $\Phi$, for $R e=0.1$ and various values of $R i$.

a diminution with this parameter, at least for the selected values of $R i$.

It is clear that the results related to heat transfer in nanofluids lead to contradictory conclusions, depending on the flow nature, thus leaving still unanswered the question if the use of nanoparticle suspensions for mixed convection applications is actually advantageous with respect to pure liquids. In fact, with dominant lid-driven flow, heat transfer enhances with nanoparticles, whereas with dominant buoyancy-driven flow the opposite effect happens, which is paradoxical when nanofluids are expected to improve heat transfer. This can be related, essentially, to the conflict between effective conductivity and viscosity with the complicity of the cavity aspect ratio, which is large and favours the effect of viscosity and disfavours that of conductivity.

\subsection{Onset of the Bicellular Flow}

In Figure 14 is depicted the evolution of $R i_{c}$, corre- 

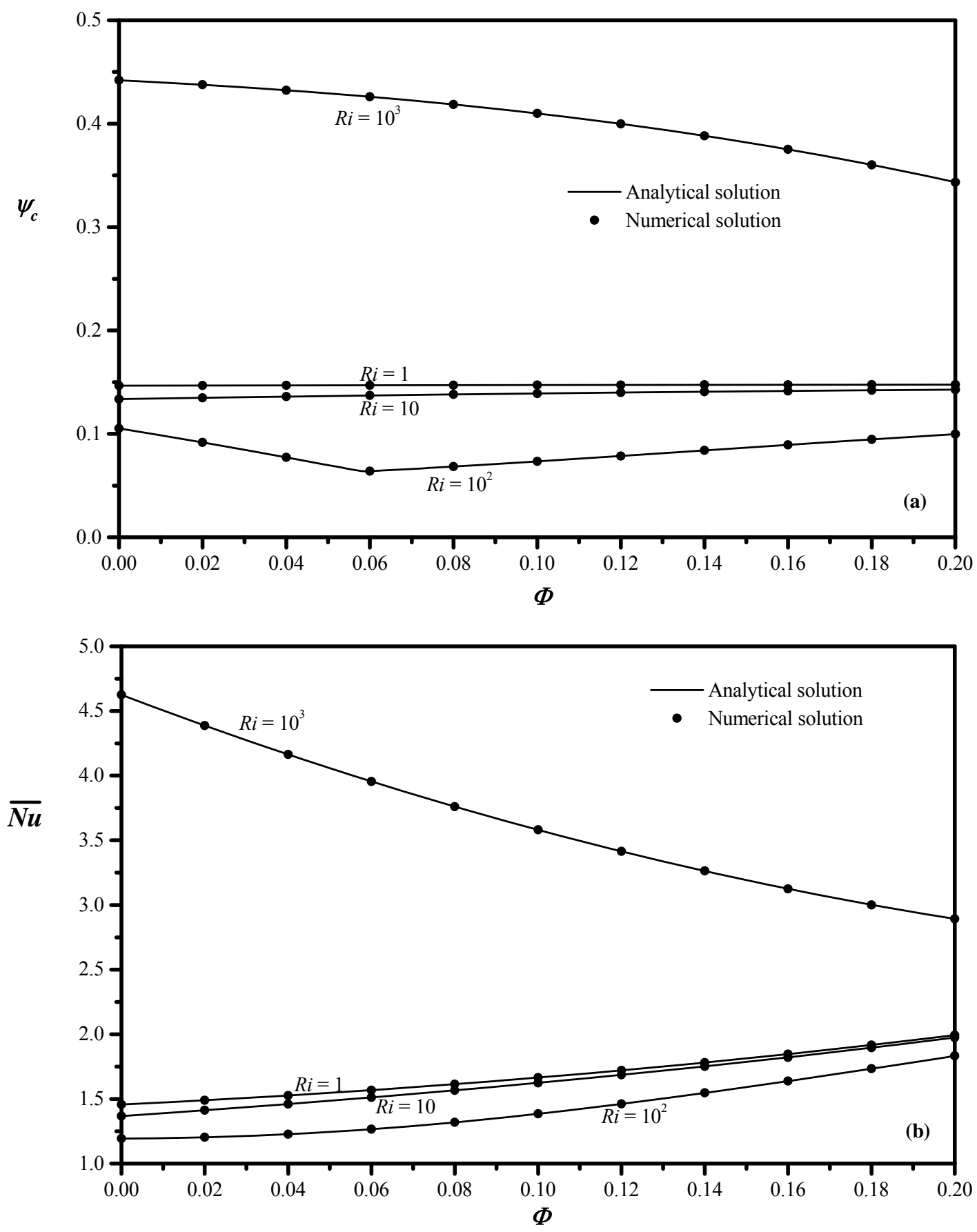

Figure 12. Flow intensity (a) and heat transfer rate (b) versus $\Phi$, for $R e=1$ and various values of $R i$.

sponding to the onset of buoyancy-driven flow, which gives rise to bicellular flow, versus $R e$, for various values of $\Phi$. As can be seen, $R i_{c}$ decreases and increases on both sides of a minimum corresponding to a particular value, $R e_{m}$, of $R e$ such that $1<R e_{m}<5$, depending on $\Phi$. This trend indicates that the appearance of the buoyancy-driven flow tends to be sometimes precocious, sometimes late around $R e_{m}$. In addition to that, the more $\Phi$ increases the more $R i_{c}$ increases, which means that the presence of nanoparticles delays the onset of buoyancy-driven flow and then opposes to the corresponding effect, confirming the deterioration observed, before, for heat transfer associated with this kind of flow.

\section{Conclusions}

In this paper, a numerical and analytical study on mixed convection in a two-dimensional horizontal shallow 

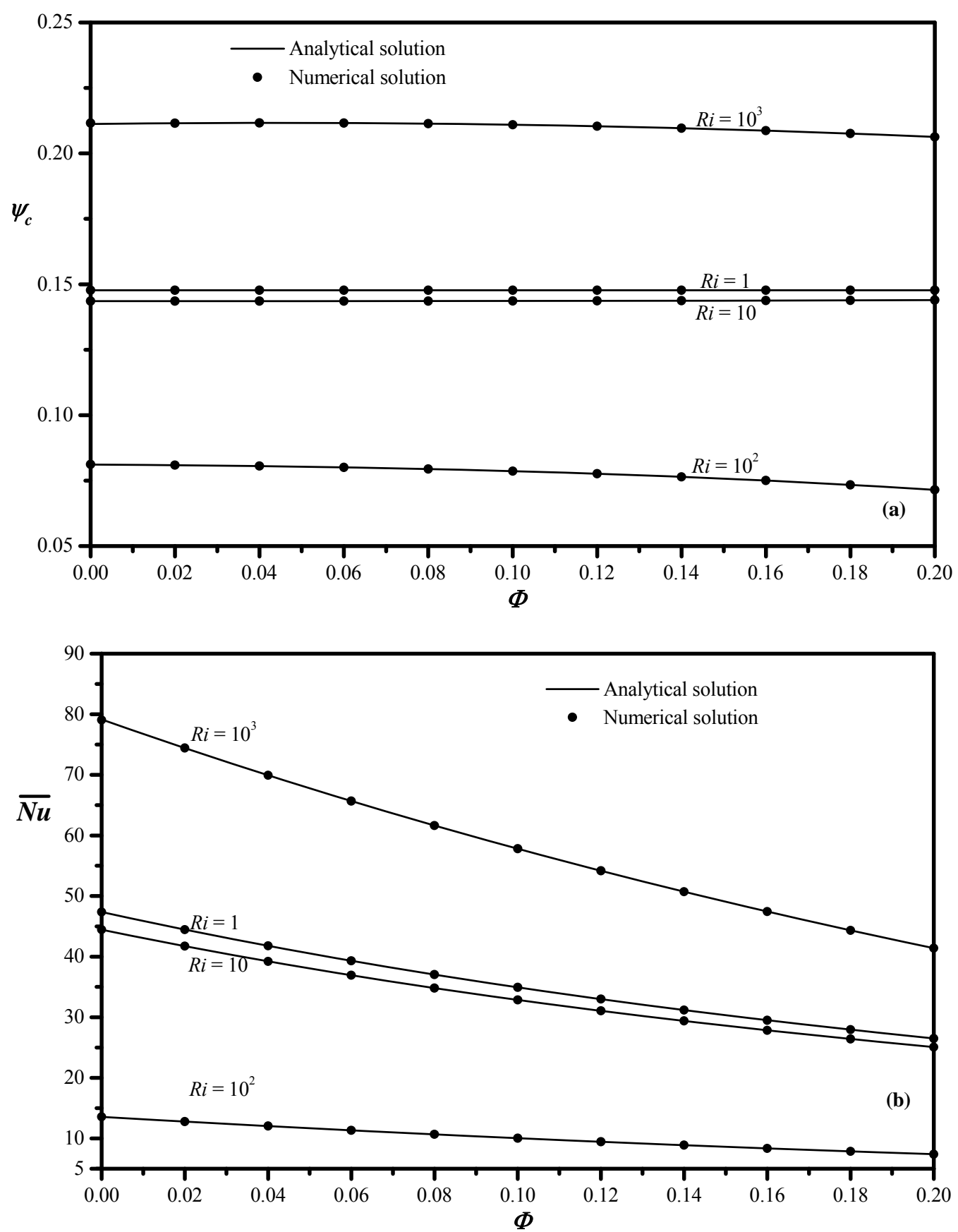

Figure 13. Flow intensity (a) and heat transfer rate (b) versus $\Phi$, for $R e=10$ and various values of $R i$.

enclosure, of aspect ratio $A=8$, filled with a nanofluid, has been conducted in the case where both short vertical sides are submitted to uniform heat fluxes while the long horizontal ones are assumed adiabatic, with the top one uniformly moving in the opposite direction to heat flux.

The full partial differential equations, governing the problem, have been solved numerically using a finite volume method. The computations, which have been limited to $C u$-water mixtures, with $\operatorname{Pr}=7$, have been carried out with governing parameters, $R e, R i$ and $\Phi$, varying, respectively, in the ranges $0.1 \leq R e \leq 10,1 \leq R i \leq 10^{3}$ and $0 \leq \Phi \leq 0.2$. Analytical solution is derived on the basis of a parallel flow assumption in the core region of the enclosure. The main findings of such an investigation can be summarized as follows:

- In the limit of the selected values of the governing parameters, analytical results, agree very well with the numerical ones, which validates mutually both the corresponding approaches.

- Flow and temperature fields strongly depend on the 


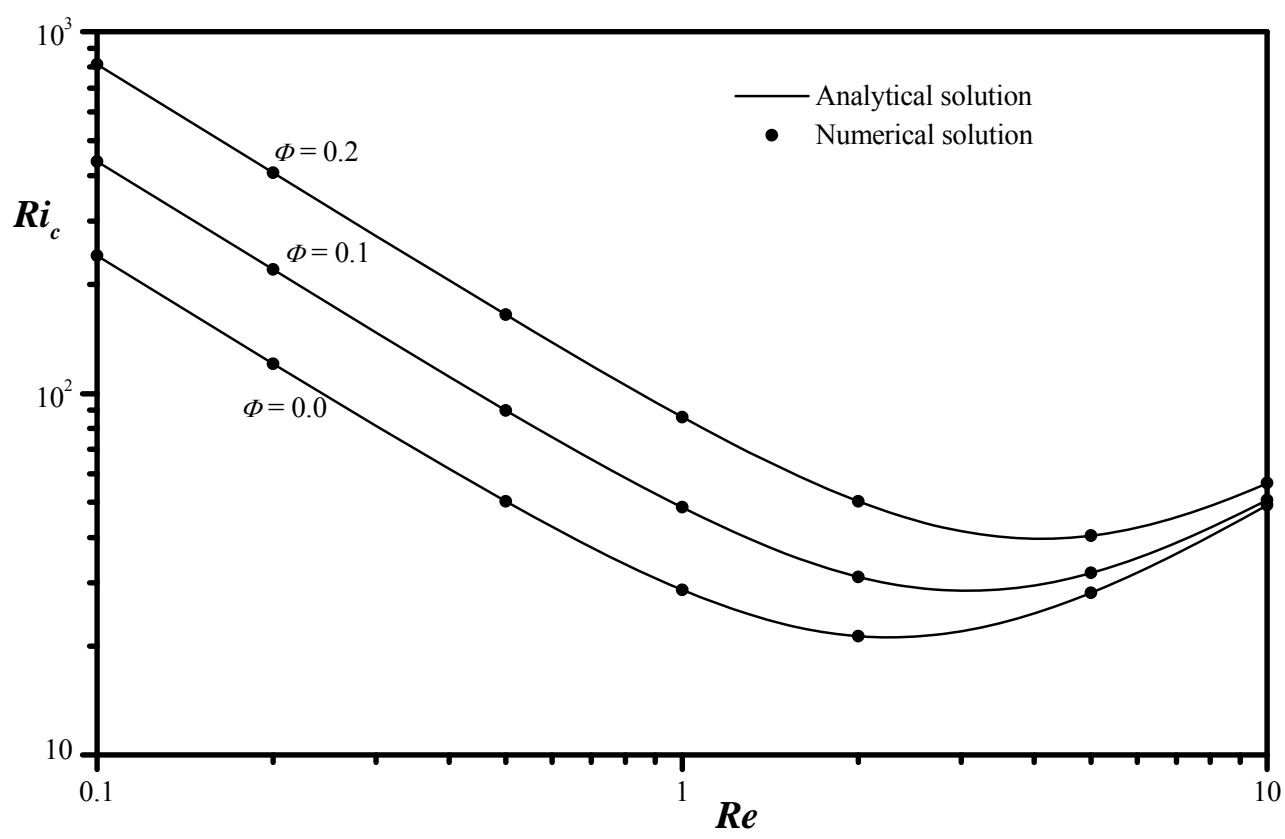

Figure 14. Limit of the onset of the bicellular flow, for various values of $\Phi$.

Richardson number, measuring the relative importance of both lid and buoyancy-driven effects.

- Increasing the Richardson number is, in general, associated with decreasing of heat transfer rate due to shear flow and increasing of that due to buoyancydriven flow.

- The addition of $\mathrm{Cu}$-nanoparticles into the pure water leads to an enhancement of lid-driven convection heat transfer.

- Against all odds, the addition of $\mathrm{Cu}$-nanoparticles into the pure water results in a degradation of buoyancydriven convection heat transfer. Therefore, although prospects of nanofluids are very promising, there is still a dearth of enough research in this area.

- The onset of buoyancy-driven flow, giving rise to bicellular flow, depends strongly on the Reynolds number and nanoparticles volume fraction.

\section{REFERENCES}

[1] S. U. S. Choi, "Enhancing Thermal Conductivity of Fluids with Nanoparticles," In: D. A. Siginer and H. P. Wang, Eds., Developments and Applications of Non-Newtonian Flows, American Society of Mechanical Engineers, New York, 1995, pp. 99-105.

[2] W. Yu, D. M. France, S. U. S. Choi and J. L. Routbort, "Review and Assessment of Nanofluid Technology for Transportation and Other Applications," Technical Report ANL/ESD/07-9, Argonne National Laboratory, Energy Systems Division, Argonne, 2007. http://www.osti.gov/bridge

[3] M. Corcione, "Heat Transfer Features of BuoyancyDriven Nanofluids inside Rectangular Enclosures Differ- entially Heated at the Sidewalls," International Journal of Thermal Sciences, Vol. 49, No. 9, 2010, pp. 1536-1546. doi:10.1016/j.ijthermalsci.2010.05.005

[4] L. A. B. Pilkington, "Review Lecture. The Float Glass Process," Proceedings of the Royal Society of London. Series A, Mathematical and Physical Sciences, Vol. 314, No. 1516, 1969, pp. 1-25. doi:10.1098/rspa.1969.0212

[5] F. J. K Ideriah, "Prediction of Turbulent Cavity Flow Driven by Buoyancy and Shear," Journal of Mechanical Engineering Sciences, Vol. 22, No. 6, 1980, pp. 287-295. doi:10.1243/JMES JOUR 1980 02205402

[6] J. Imberger and P. F. Hamblin, "Dynamics of Lakes, Reservoirs, and Cooling Ponds," Annual Review of Fluid Mechanics, Vol. 14, 1982, pp. 153-187. doi:10.1146/annurev.fl.14.010182.001101

[7] C. K. Cha and Y. Jaluria, "Recirculating Mixed Convection Flow for Energy Extraction," International Journal of Heat Mass Transfer, Vol. 27, No. 10, 1984, pp. 1801-1810. doi:10.1016/0017-9310(84)90162-5

[8] R. K. Tiwari and M. K. Das, "Heat Transfer Augmentation in a Two-Sided Lid-Driven Differentially Heated Square Cavity Utilizing Nanofluids," International Journal of Heat and Mass Transfer, Vol. 50, No. 9-10, 2007, pp. 2002-2018.

doi:10.1016/j.ijheatmasstransfer.2006.09.034

[9] E. Abu-Nada and A. J. Chamkha, "Mixed Convection Flow in a Lid-Driven Square Enclosure Filled with a Nanofluid," European Journal of Mechanic B/Fluids, Vol. 29 , No. 6, 2010, pp. 472-482. doi:10.1016/j.euromechflu.2010.06.008

[10] M. Mahmoodi, "Mixed Convection inside Nanofluid Filled Rectangular Enclosures with Moving Bottom Wall," International Journal of Thermal Sciences, Vol. 15, No. 3, 2011, pp. 889-903. 


\section{doi:10.2298/TSCI101129030M}

[11] M. A. Mansour, R. A. Mohamed, M. M. Abd-Elaziz and S. E. Ahmed, "Numerical Simulation of Mixed Convection Flows in a Square Lid-Driven Cavity Partially Heated from below Using Nanofluid," International Communication in Heat and Mass Transfer, Vol. 37, No. 10, 2010, pp. 1504-1512.

doi:10.1016/j.icheatmasstransfer.2010.09.004

[12] M. Muthtamilselvan, P. Kandaswamy and J. Lee, "Heat Transfer Enhancement of Copper-Water Nanofluids in a Lid-Driven Enclosure," Communications in Nonlinear Science and Numerical Simulation, Vol. 15, No. 6, 2010, pp. 1501-1510. doi:10.1016/j.cnsns.2009.06.015

[13] H. Nemati, M. Farhadi, K. Sedighi, E. Fattahi and A. A. R. Darzi, "Lattice Boltzmann Simulation of Nanofluid in Lid-Driven Cavity," International Communication in Heat and Mass Transfer, Vol. 37, No. 10, 2010, pp. 15281534. doi:10.1016/j.icheatmasstransfer.2010.08.004

[14] F. Talebi, A. H. Mahmoudi and M. Shahi, "Numerical Study of Mixed Convection Flows in a Square LidDriven Cavity Utilizing Nanofluid," International Communication in Heat and Mass Transfer, Vol. 37, No. 1, 2010, pp. 79-90. doi:10.1016/j.icheatmasstransfer.2009.08.013

[15] G. A. Sheikhzadeh, N. Hajialigol, M. E. Qomi and A. Fattahi, "Laminar Mixed Convection of Cu-Water Nanofluid in Two Sided Lid-Driven Enclosures," Journal of Nanostructures, Vol. 1, No. 1, 2012, pp. 44-53.

[16] M. A. Karimipour and M. M. Bazofti, "Periodic Mixed Convection of a Nanofluid in a Cavity with Top Lid Si- nusoidal Motion," International Journal of Mechanical and Materials Engineering, Vol. 1, No. 1, 2010, pp. 3439.

[17] Y. Xuan and W. Roetzel, "Conceptions for Heat Transfer Correlation of Nanofluids," International Journal of Heat and Mass Transfer, Vol. 43, No. 19, 2000, pp. 3701-3707. doi:10.1016/S0017-9310(99)00369-5

[18] E. B. Ogut, "Natural Convection of Water-Based Nanofluids in an Inclined Enclosure with a Heat Source," International Journal of Thermal Sciences, Vol. 48, No. 11, 2009, pp. 2063-2073. doi:10.1016/j.ijthermalsci.2009.03.014

[19] M. Lamsaadi, M. Naimi and M. Hasnaoui, "Natural Convection Heat Transfer in Shallow Horizontal Rectangular Enclosures Uniformly Heated from the Side and Filled with Non-Newtonian Power Law Fluids," Energy Conversion and Management, Vol. 47, No. 15-16, 2006, pp. 2535-2551. doi:10.1016/j.enconman.2005.10.028

[20] S. Patankar, "Numerical Heat Transfer and Fluid Flow," Hemisphere, Washington DC, 1980.

[21] A. Bejan, "The Boundary Layer Regime in a Porous Layer with Uniform Heat Flux from Side," International Journal of Heat Mass Transfer, Vol. 26, No. 9, 1983, pp. 1339-1346. doi:10.1016/S0017-9310(83)80065-9

[22] E. Abu-Nada, Z. Masoud and A. Hijazi, "Natural Convection Heat Transfer Enhancement in Horizontal Concentric Annuli Using Nanofluids," International Communication in Heat and Mass Transfer, Vol. 35, No. 5, 2008, pp. 657-665. doi:10.1016/j.icheatmasstransfer.2007.11.004 